\title{
Nonlinear Dynamics of Human Aortas for Material Characterization
}

\author{
Marco Amabiliø, ${ }^{1,2, *}$ Prabakaran Balasubramanian, ${ }^{1}$ Isabella Bozzo, ${ }^{1,3}$ Ivan D. Breslavsky, ${ }^{1}$ \\ Giovanni Ferrari, ${ }^{1}$ Giulio Franchini, ${ }^{1}$ Francesco Giovanniello, ${ }^{1}$ and Chloé Pogue ${ }^{1}$ \\ ${ }^{1}$ Department of Mechanical Engineering, McGill University, \\ 817 Sherbrooke St. W, Montreal, Quebec, Canada, H3A OC3 \\ ${ }^{2}$ Department of Biomedical Engineering, McGill University, \\ 817 Sherbrooke St. W, Montreal, Quebec, Canada, H3A OC3 \\ ${ }^{3}$ Faculty of Medicine, McGill University, 3605 de la Montagne, Montreal, Quebec, Canada, H3G 2 MI
}

(Received 28 June 2019; revised manuscript received 26 October 2019; published 23 January 2020)

\begin{abstract}
Evaluating the nonlinear dynamics of human descending thoracic aortas is essential for building the next generation of vascular prostheses. This study characterizes the nonlinear dynamics, viscoelastic material properties, and fluid-structure interaction of 11 ex-vivo human descending thoracic aortas the full range of physiological heart rates. The aortic segments are harvested from heart-beating donors screened for transplants. A mock circulatory loop is developed to reproduce physiological pulsatile pressure and flow. The results show cyclic axisymmetric diameter changes, which are satisfactorily compared to in-vivo measurements at a resting pulse rate of $60 \mathrm{bpm}$, with an additional bending vibration. An increase of the dynamic stiffness (i.e., storage modulus) with age is also observed. This increase is accompanied by a strong reduction with age of the cyclic diameter change during the heart pulsation at $60 \mathrm{bpm}$ and by a significant reduction of the loss factor (i.e., damping). Large dissipation is observed at higher pulse rates due to the combined effects of fluid-structure interaction and viscoelasticity of the aortic wall. This study presents data necessary for developing innovative grafts that better mimic the dynamics of the aorta.

DOI: $10.1103 /$ PhysRevX.10.011015

Subject Areas: Biological Physics, Materials Science, Medical Physics
\end{abstract}

\section{INTRODUCTION}

Cardiovascular disease, the leading cause of death in the United States, is projected to cost the nation \$1.1 trillion dollars by 2035 [1]. An escalating interest in cardiovascular surgery involves replacing widely used synthetic grafts, like Dacron, with innovative biomaterials or tissue engineering [2], as it fails to correctly mimic the dynamic behavior of the aorta, negatively affecting the local blood pressure and flow through a large reduction of the Windkessel effect [3-5]. Complications like heart overloading and replacement surgeries may ensue from prolonged usage of synthetic grafts, thus inciting research in understanding aortic tissue behavior to design mechanically compatible grafts [6].

The region of interest is the descending thoracic aorta that can be subjected to fatal aneurysms and acute

Corresponding Author.

marco.amabili@mcgill.ca

Published by the American Physical Society under the terms of the Creative Commons Attribution 4.0 International license. Further distribution of this work must maintain attribution to the author(s) and the published article's title, journal citation, and DOI. dissection. It is located distal to the aortic arch and resembles a tapering circular cylindrical shape. The proximal diameter in-vivo ranges from 22.8 to $26.6 \mathrm{~mm}$, and the distal diameter ranges from 21.1 to $24.5 \mathrm{~mm}$ [7]. A resting heart rate of 60 beats per minute (bpm) corresponds to a mean reference pressure of $100 \mathrm{~mm} \mathrm{Hg}$ with a $40-\mathrm{mm} \mathrm{Hg}$ pulse pressure amplitude variation for the aorta [8]. The Windkessel effect describes a cyclic change of the aorta's diameter that creates cyclic volume changes within the aortic segment. The cyclic diameter change, in response to the pulsatile flow in-vivo, varies from $2.7 \%$ to $8 \%$, with younger patients exhibiting larger expansion [7]. It is determined that aortic walls stiffen with age [9].

Mock circulatory loops (MCL) were developed to test cardiovascular devices in vitro by recreating physiological pulsatile blood flow conditions [10-12]. The principal components of closed-loop MCL are as follows: (i) the pulsatile pump, (ii) the expansion chamber with an air cushion to replicate the Windkessel effect of the arterial tree, and (iii) pinch valves to reproduce the peripheral resistance. To date, tests on eleven Merino sheep descending thoracic aortas at 108 and $110 \mathrm{bpm}[13,14]$, seven human common carotid arteries at $70 \mathrm{bpm}$ [15], and canine descending thoracic aortas with vasoactive-agent smoothmuscle activation [16], as reported in the literature. To the 
authors' knowledge, MCLs were not used for the dynamic characterization of human aortas. These data are necessary to develop nonlinear viscoelastic material models of the human aorta.

A specific nonlinear viscoelastic model with internal variables is applied to the human aorta $[17,18]$. Improved viscoelastic models require a thorough understanding of the relationship between the viscoelasticity and the microstructure of the aorta. The aorta consists of a threedimensional network of intertwined fibers, including collagen and elastin [19]. Fiber contributions to the relaxation process are material specific; they depend on the intrinsic viscoelasticity of the fibers and the rearrangement of the network resulting from different fibers relaxing at nonuniform rates [19].

Furthermore, the aorta consists of three layers-the intima, the media, and adventitia - with different compositions and hyperelastic material properties related to their function [9,20,21]. Traditional histology [22] and nonlinear laser scanning microscopy with second harmonic generation [21] have led to detailed microstructural models of the collagen fibers accounting for directions and dispersion. This work has resulted in a sophisticated layer-specific hyperelastic material model considering the microstructure [23]. A layer-specific hyperelastic characterization of coronary arteries is also available [24]. However, viscoelastic models of the human aorta are less developed, despite experiments indicating that the dynamic storage modulus is significantly larger than the static modulus. Few experiments, all without the use of vasoactive agents, have been performed to determine the loss tangents and storage moduli of human aortas [9,25-27]. Layer-specific viscoelastic properties were found to increase from static values by 1.2 to well over 2 times, with the loss tangent of the media in the circumferential direction varying from 0.06 to 0.21 at $180 \mathrm{bpm}$, for small-amplitude cyclic strain [9]. One study using harmonic volumetric inflation of human aortic segments with a syringe found that the loss tangent grew with frequency, ranging from 0.079 to 0.15 [25]. Fifteen abdominal aortas were tested in-vivo at rest (specifically around $72 \mathrm{bpm}$ ), and, assuming a ratio of 5.3 between the mean aortic radius and the wall thickness, an average loss tangent of 0.12 and storage modulus of $604 \mathrm{kPa}$ were obtained [27]. Four healthy human abdominal aortas were shear tested at low strain with a rotational rheometer, reporting loss tangent values from 0.04 to 0.1 and storage moduli gradually increasing with frequency [26]. However, these values were nonphysiological as the applied prestretch was only $1.5 \%$ [28].

This study aims to characterize the nonlinear viscoelastic properties and the nonlinear dynamics of human descending thoracic aortas. Eleven aortas from transplant-grade donors were tested ex-vivo in a MCL developed to measure the dynamic displacements of the aortic wall under a pulsatile flow. The loop was tuned to accurately reproduce physiological flow and pressure at different pulse rates, covering the full range of physiological heart rates.

\section{MCL CONCEPTION AND DYNAMIC ANALYSIS}

\section{A. Aorta retrieval and preparation}

The aortas were obtained from Transplant Québec, the provincial donation coordination center. The research was approved by the ethical committee of McGill University. The donors were sustained on a respirator until the explant procedure. This process ensured the organs and tissues harvested were viable without signs of necrosis. The positive screening for transplant of the patient guaranteed no known diseases or conditions that compromised the aorta. The aortas were excised by cardiothoracic surgeons while retrieving the donor organs for transplant. The aortic specimens were transported at $4^{\circ} \mathrm{C}$ in a saline solution $(0.9 \% \mathrm{w} / \mathrm{v}$ of $\mathrm{NaCl})$ in transplant storage containers.

All specimens were tested in the MCL within 24 hours to minimize the effect of decomposition on the structural integrity. The aortas were stored in a physiological saline solution $(0.9 \% \mathrm{w} / \mathrm{v}$ of $\mathrm{NaCl})$ and refrigerated at $4{ }^{\circ} \mathrm{C}$ upon arrival to the test lab, up to the onset of the experiments. The aortic material also underwent quasistatic and dynamic uniaxial tests on dissected strips of intima, media, and adventitia layers within the subsequent 48 hours [9]. Finally, slices were prepared for histology and nonlinear laser scanning microscopy imaging.

Upon reception of the aortas, a standardized preparation was performed. All samples underwent the same debridement, consisting of the removal of the periaortic adipose and connective tissue and of the protruding portion of the arterial branches. Next, the aortic arch at the proximal end was eliminated, and all the holes were sealed. The proximal and distal external diameters were measured with scaled photographs captured with a Canon EOS 70D digital camera. The camera projected parallel to the circumferential surface using a tripod. A tapered cylindrical tube was the approximate geometry of the descending thoracic aorta segment prepared for the MCL experiment, with the proximal end larger than the distal one. The thickness of the aorta wall was measured at several points along the length of the specimen with a laser sensor (optoNCDT Micro-Epsilon) after the experiment.

\section{B. MCL design}

A custom MCL was built to study the nonlinear dynamics of human descending thoracic aortas. Once the preparation was completed, the aortic segment was fixed to rigid cylindrical supports whose diameters were about the size of the inner diameter of the specimen. Sets of supports were manufactured to accommodate the varying diameters of each donor. A ring of Dacron was wrapped around each of the proximal and distal ends, before fixating the 


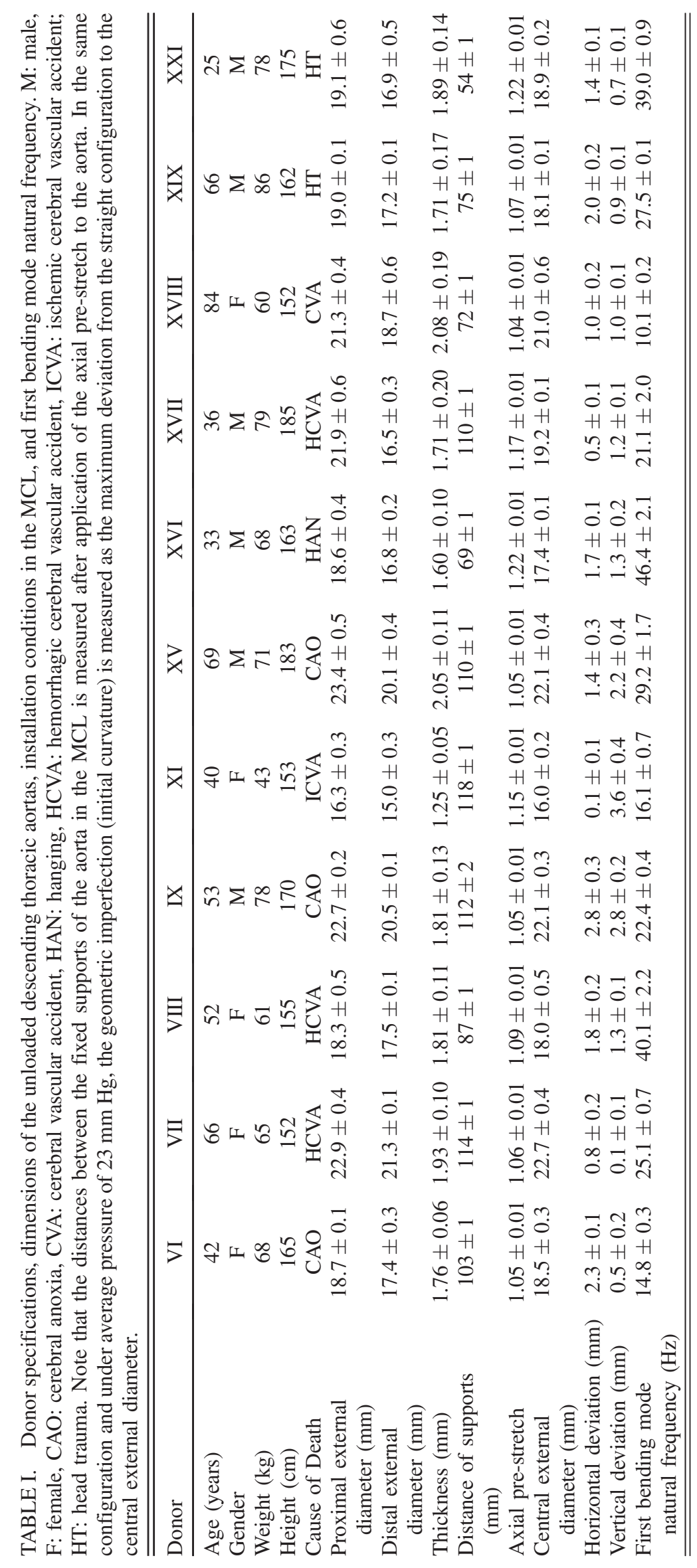


specimen with hose clamps to avoid damaging the tissue. The proximal end was attached to the inlet and the distal end to the outlet. The distance between the two clamps was measured with a Vernier caliper, which gave the free length of the aorta. Then, an axial prestretch was applied in correspondence with the donor age by adjusting the separation distance of the mounts supporting the clamps; smaller stretches were used for older donors [28,29]. The prestretch values are given in Table I.

The boundary condition of zero axial displacement at the ends well reproduces the natural behavior of the aortic segment in the human body, which is not elongating during pulsation; the condition of zero lateral displacement at the ends, which affects only bending, approximates the natural behavior. This result potentially introduces less dissipation than a moving connection with the remaining part of the aorta. Aortic branches and periaortic connective tissue can also introduce additional dissipation, especially for bending vibrations.

The aorta was wrapped in gauze soaked in physiological saline solution $(0.9 \% \mathrm{w} / \mathrm{v}$ of $\mathrm{NaCl})$. This process preserves the moisture in the tissue and prevent it from drying up. The gauze was removed during the active measurement recordings. The geometric imperfection, i.e., the initial curvature, of the prestretched aorta assembled in the MCL was measured as the maximum deviation from the straight configuration by scaled digital photography.

The MCL, shown in Fig. 1, aimed to reproduce the pulsatile pumping of the heart, the Windkessel effect, and peripheral resistance of the portion of the arterial tree. The loop was built with $3 / 4$ " ID Tygon tubing leading to the tank; a larger tube was used for the return to the pump. To simulate the compliance of the arterial tree upstream and downstream of the aorta, two expansion chambers (manufactured by BDC Laboratories) were placed before and after the aorta. Each chamber contained a volume of approximately 2.25 liters. The system was filled with physiological saline solution $(0.9 \% \mathrm{w} / \mathrm{v}$ of $\mathrm{NaCl}) \mathrm{kept}$ at a temperature of $37^{\circ} \pm 0.1^{\circ} \mathrm{C}$ by a controlled heater. The saline solution had a dynamic viscosity of $0.69 \mathrm{mPa} \mathrm{s}$ at $37^{\circ} \mathrm{C}$. Tests were also performed with a mixture (in volume) of $55 \%$ saline solution and $45 \%$ glycerin, with a dynamic viscosity of $3.5 \mathrm{mPa} \mathrm{s}$ at $37^{\circ} \mathrm{C}$, which is the same as blood in large arteries [30]. The mass density of the mixture was $1119 \mathrm{~kg} \mathrm{~m}^{3}$ versus $1050 \mathrm{~kg} \mathrm{~m}^{3}$, which is the density of blood. If not otherwise specified, the results presented were obtained with the saline solution. A tank, open to the atmospheric pressure, was installed to separate the test section from the suction of the inlet of the pump; it replicates the separation of the arterial tree from the venous system.

Air pockets were trapped on top of each of the expansion chambers. The trapped air provided compliance since air is compressible, thus functioning as a pneumatic spring. The spring stiffness was proportional to the volume of the trapped air, which was adjusted with pneumatic valves. A gauge measured the pressure of the air pocket, which varied during the flow cycle. Downstream of the aorta, a pinch valve was installed to simulate the arterial resistance to flow. The tuning of the air volume in the expansion chambers and the level of tube squeezing introduced by the pinch valve allowed us to obtain the desired $100-\mathrm{mm} \mathrm{Hg}$ mean pressure, with a diastolic pressure of about $80 \mathrm{~mm} \mathrm{Hg}$ and a systolic pressure of around $120 \mathrm{~mm} \mathrm{Hg}$. The difference of systolic and diastolic pressures is referred to as the pulse pressure. The tuning of the air volumes had to be repeated at every change of the simulated heart rate (pulse rate).

The pulsatile flow was produced by a model 55-3305 piston pump from Harvard Apparatus. The pump had ball check valves, stroke (range of 15 to $100 \mathrm{~cm}^{3}$ ), systole/ diastole duration ratio, and pulse rate (bpm) controls. The maximum pulse rate of the original Harvard Apparatus pump was $100 \mathrm{bpm}$. In this case, the original Bodine 6126 electric motor of the pump was substituted with a Bodine 6125 motor capable of about double the speed. This pump allowed us to reproduce the full range of physiological heart rates. Similar mean systolic and diastolic pressures were maintained at different pulse rates to ensure that similar pressure loads were acting on the aorta. Two Carolina Medical Electronics magnetic flowmeters (model FM501) with flow probes (model EP690), and two Millar catheter pressure sensors SPR-524 (size 3.5 F) with pressure signal conditioners PCU-2000 were placed at the inlet and outlet of the aorta. The typical maximum measurement error for the Millar catheter pressure sensor was $1.5 \mathrm{~mm} \mathrm{Hg}$, and for the Carolina Medical magnetic flowmeter, it was 0.3 liters/ min.

A schematic of the mock circulatory loop designed for this study is shown in Fig. 1(a), indicating the Harvard Apparatus pump customized for increased pulse rate, the two expansion chambers, the test section, the tuning clamp valve, and the water tank with temperature control.

A photograph of the test section in Fig. 1(b) shows the attachment of the aorta to the supports, and the flowmeter and pressure transducers. A schematic of the axisymmetric and bending modes, with the corresponding laser setup, used to capture the displacement in the respective directions, is shown in Fig. 1(c). The axisymmetric breathing mode indicates a radial expansion or contraction, as in Fig. 1(d), and the bending mode refers to a cyclic oscillation of the longitudinal axis of the aorta without deformation of the cross section, as in Fig. 1(e).

\section{Dynamic displacement measurement}

Four Polytec laser Doppler vibrometers (model OFV 505/PSV 500) equipped with displacement decoders were installed at 90 degrees from one another along the circumference of the aorta specimen. They were pointing at the mid-length of the aortic segment, as shown in Fig. 1(a). 


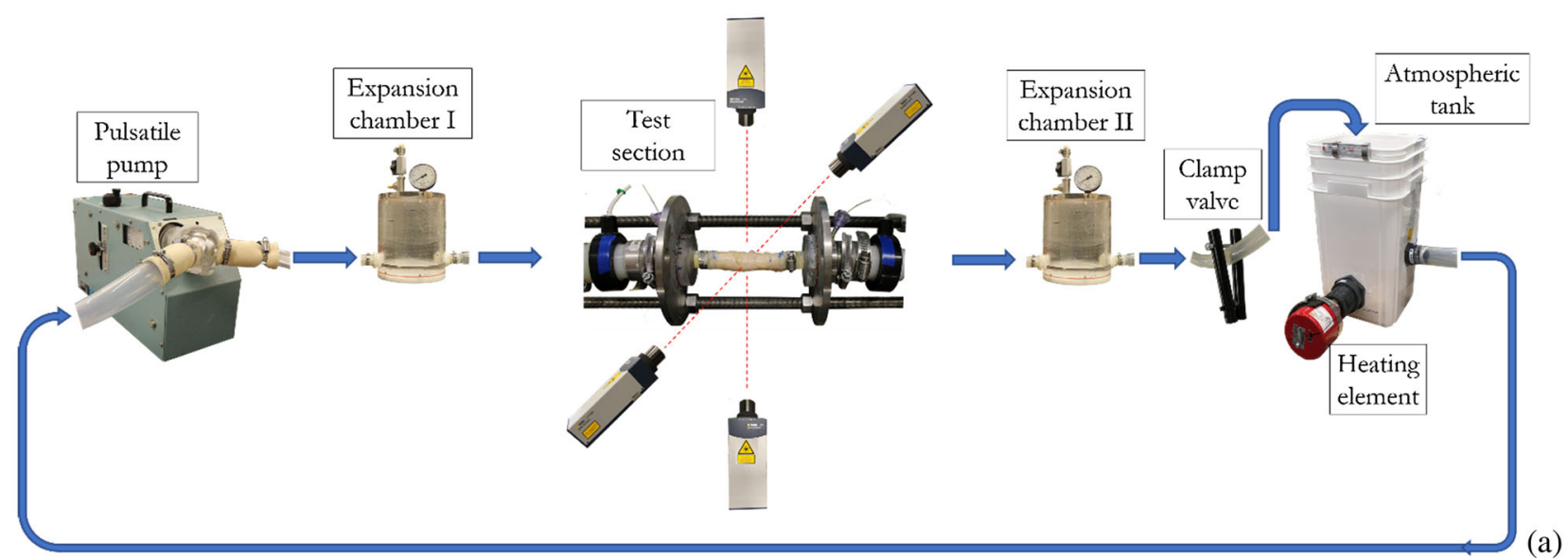

(a)

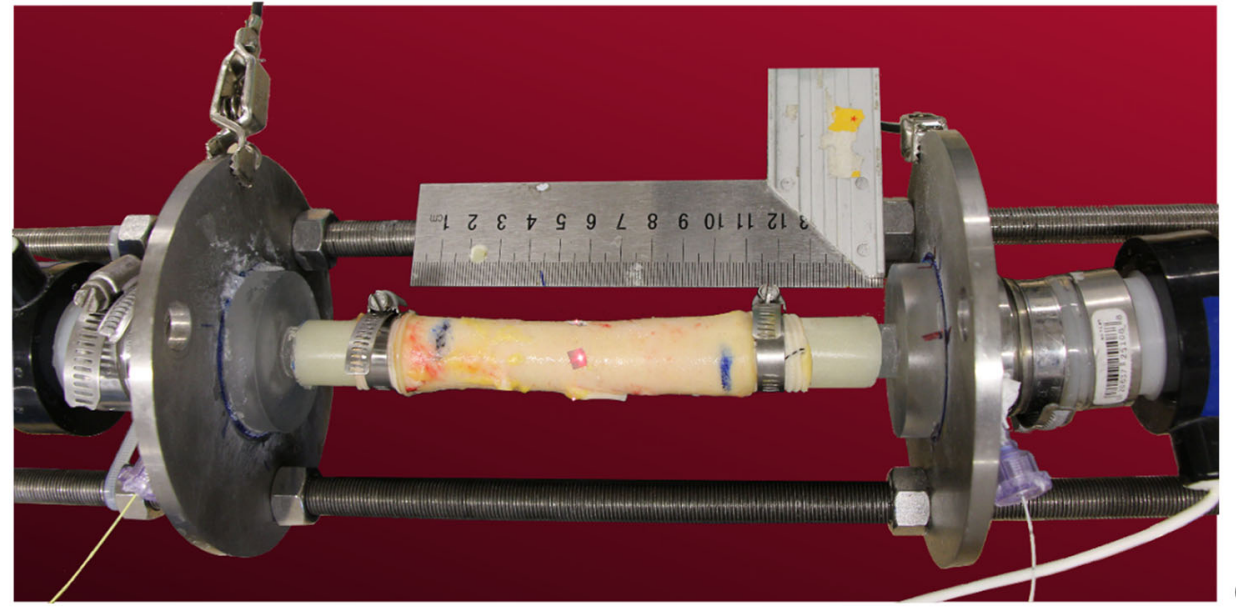

(b)
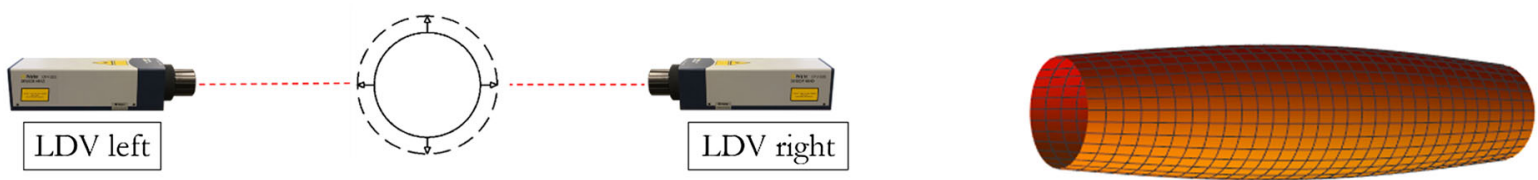

(d)
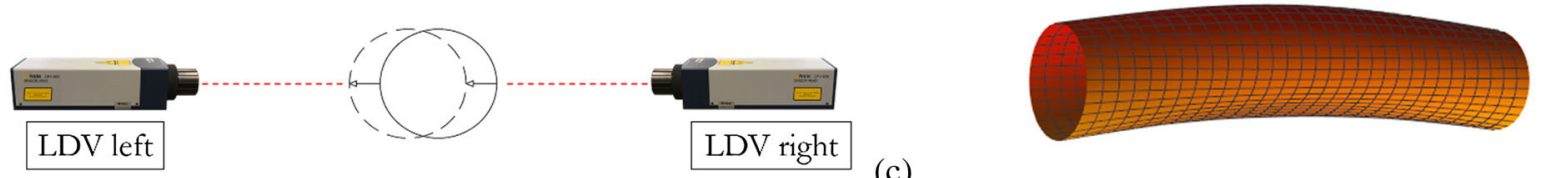

(c)

(e)

FIG. 1. Experimental setup with dynamic measurement schematic: (a) scheme of the MCL: Harvard Apparatus volumetric pump, expansion chamber, test section, second expansion chamber, pinch valve, atmospheric tank with thermal heater and temperature control; (b) test section of the MCL with the assembled aorta from donor VII, two flowmeters and two catheter pressure gauges (the thin wires coming out from them are clearly visible); the reflecting tapes with laser spots are visible; (c) top: cyclic change of the aortic diameter, shown in a cross-section, measured by two aligned laser Doppler vibrometers (LDV), bottom: bending oscillation of the aorta, shown in a cross-section, measured by two aligned LDV; (d) axisymmetric diameter change of the aortic segment; (e) bending oscillation of the aortic segment.

Small coupons of thin reflective tape were stuck with cyanoacrylate glue directly to the aorta at the four laser target locations. Each vibrometer measured the dynamic displacement in the direction of the laser beam, capturing a signal caused by the simultaneous diameter change and bending oscillation. Because of the cyclic diameter change, the aorta cross section remains circular at each given instant, while the radius changes with time and the aortic center does not move. For pure cyclic diameter changes, the two opposite lasers would measure identical signals both in phase and amplitude at any given instant. Instead, for bending oscillation, the radius is constant, but the location of the center of the aortic cross section varies. For pure bending oscillations, one pair of laser sensors, placed on 
opposite sides of the aortic cross section, would measure signals identical in amplitude but with opposite phases.

The four laser signals are denoted as $V_{\text {top }}$ (top), $V_{\text {bot }}$ (bottom), $V_{\text {left }}$ (left horizontal), and $V_{\text {right }}$ (right horizontal). The amplitudes of the dynamic change of diameter in the vertical and horizontal directions at the mid-length of the aorta are

$$
\begin{aligned}
& \Delta d_{\text {vert }}=V_{\text {top }}+V_{\text {bot }}, \\
& \Delta d_{\text {hor }}=V_{\text {left }}+V_{\text {right }},
\end{aligned}
$$

respectively. Here, the two orthogonal directions, horizontal and vertical, are with respect to the aorta position in the MCL, and both belong to the plane orthogonal to the aorta's longitudinal axis. For a circular and straight aorta without imperfections, the two diameter changes should be equivalent. Differences between the two measurements can be introduced by imperfections. The two bending components of the oscillation in the vertical and horizontal directions are

$$
\begin{gathered}
\Delta b_{\text {vert }}=\left(V_{\text {top }}-V_{\text {bot }}\right) / 2, \\
\Delta b_{\text {hor }}=\left(V_{\text {left }}-V_{\text {right }}\right) / 2,
\end{gathered}
$$

respectively. A diagram depicting the laser measurements of dynamic diameter change and bending oscillation is presented in Fig. 1(c). The absolute measurement error of the dynamic displacement incurred by the laser Doppler vibrometers is $4 \mu \mathrm{m}$. This value is mainly attributed to alignment errors. All the signals were recorded and processed through the LMS Test.Lab software and the high-performance SCADAS front-end, using dc inputs to avoid any filter distortion.

\section{Donor and vessel specifications}

The aortas tested for this study were received between Fall 2017 and Fall 2019. The patient specifications (age, gender, weight, and cause of death) and the geometry of the aortas (proximal, distal, and central diameters, thickness, lengths, and horizontal and vertical deviations) are summarized in Table I. Eleven donors of both genders, with an average age of $51.5 \pm 17.3$ years, were selected for the study. The nomenclature system identifying the donors (VI-XXI) was chosen for consistency with the material characterization study performed sharing some specimens [9].

A descending thoracic aorta prepared for the MCL experiment is shown in Fig. 2. This figure highlights the portion of the aorta that was selected for testing. Also presented is a photograph of the collagen fibers in a cross section of the aorta from donor VI, obtained by nonlinear laser scanning microscopy with second-harmonic generation. It demonstrates the distinct separation of the three
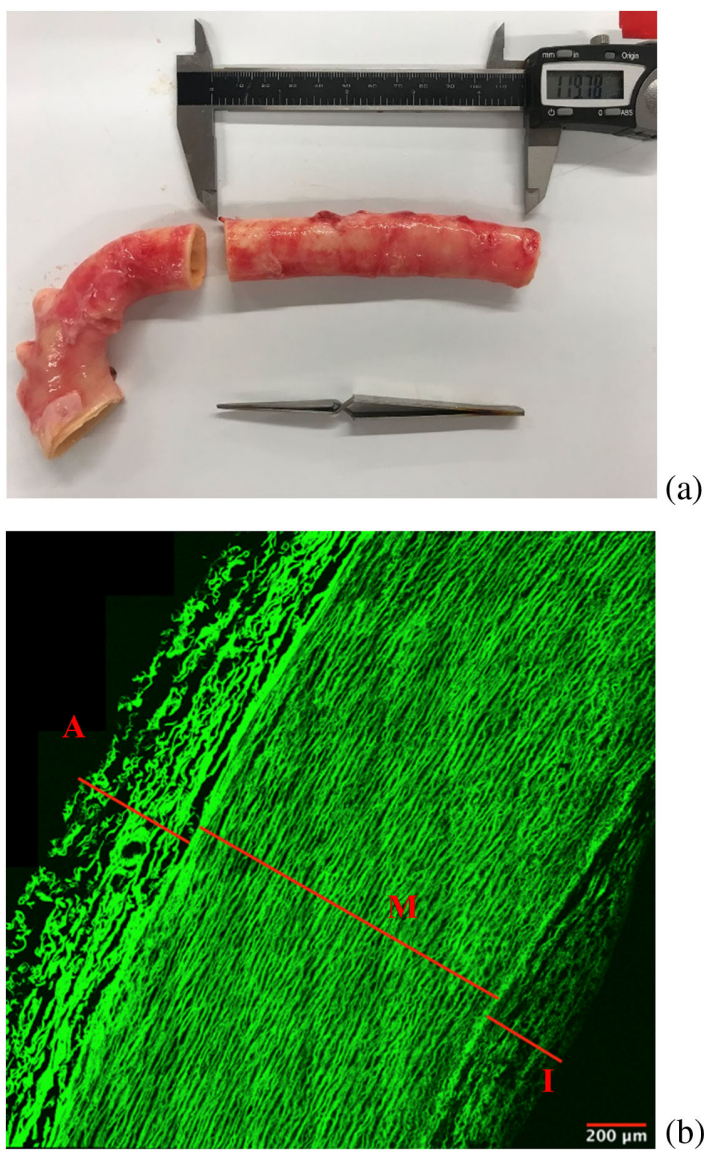

(b)

FIG. 2. Specimen preparation and microscopy imaging: (a) thoracic aorta from donor IX: aortic arch separated from the descending thoracic aorta; the aortic branches and part of the periaortic connective tissue were previously removed; (b) image of the cross-section of the aorta from donor VI, $20 \mu \mathrm{m}$ thickness, obtained by nonlinear laser scanning microscopy with secondharmonic generation to show collagen fibers. I: intima, M: media; A: adventitia.

layers of the aorta. Understanding the direction and dispersion of the collagen fibers, for the three layers, is important for building accurate viscoelastic material models. The scan indicates that the collagen fibers are well aligned in this section; they are more scattered in the adventitia, highly ordered in the media, and less prevalent in the intima.

\section{TIME AND FREQUENCY DOMAIN RESULTS}

\section{A. Time domain measurements}

The experiments in the MCL were performed at different pulse rates, from roughly $60 \mathrm{bpm}$ to $180 \mathrm{bpm}$ (a rate around 20 or $30 \mathrm{bpm}$ was also measured for some cases). The dynamic response was captured with four laser Doppler vibrometers at $90^{\circ}$ from each other and pointing radially at midlength of the aortic segment. The outlet flow rate and the inlet and outlet pressures were measured; they were also used as control parameters to tune the MCL and ensure that 
the experiments were conducted in the physiological range. A volumetric stroke of $45 \mathrm{~cm}^{3}$ (64\% of the volume of $70 \mathrm{~cm}^{3}$ pumped by the heart reaches the descending thoracic aorta [31], which gives about $45 \mathrm{~cm}^{3}$ ) was used. The dynamic responses of the aortic segment from donor $\mathrm{XI}$ at 60, 125, and $170 \mathrm{bpm}$ are presented in Fig. 3 (data for pulse rates 80, 100, and $150 \mathrm{bpm}$ are given in the Appendix A). The cyclic pressure and flow variations, and the diameter change (axisymmetric displacement) and bending displacement are shown with respect to time for three periods. The pressure was bound between the physiological limits of $80 \mathrm{~mm} \mathrm{Hg}$ and $120 \mathrm{~mm} \mathrm{Hg}$ within a tolerance (e.g., $\pm 5 \mathrm{~mm} \mathrm{Hg}$ ) since it was adjusted by tuning the MCL components: the size of the air cushion in the expansion chambers and the regulation of the clamp valve. The dynamic responses varied with the pulse rate. An increase in systolic pressure due to aging was reported in the elderly [7]. In this study, the fixed mean pressure and the pulse pressure were used to reproduce physiological conditions in order to apply similar dynamic pressure loads that would allow one to easily compare aortic responses of different donors.

At $60 \mathrm{bpm}$, the inlet and outlet pressures were almost synchronous and identical, which indicates that the pressure drop was small and the MCL was tuned to avoid producing destructive reflecting pressure waves while
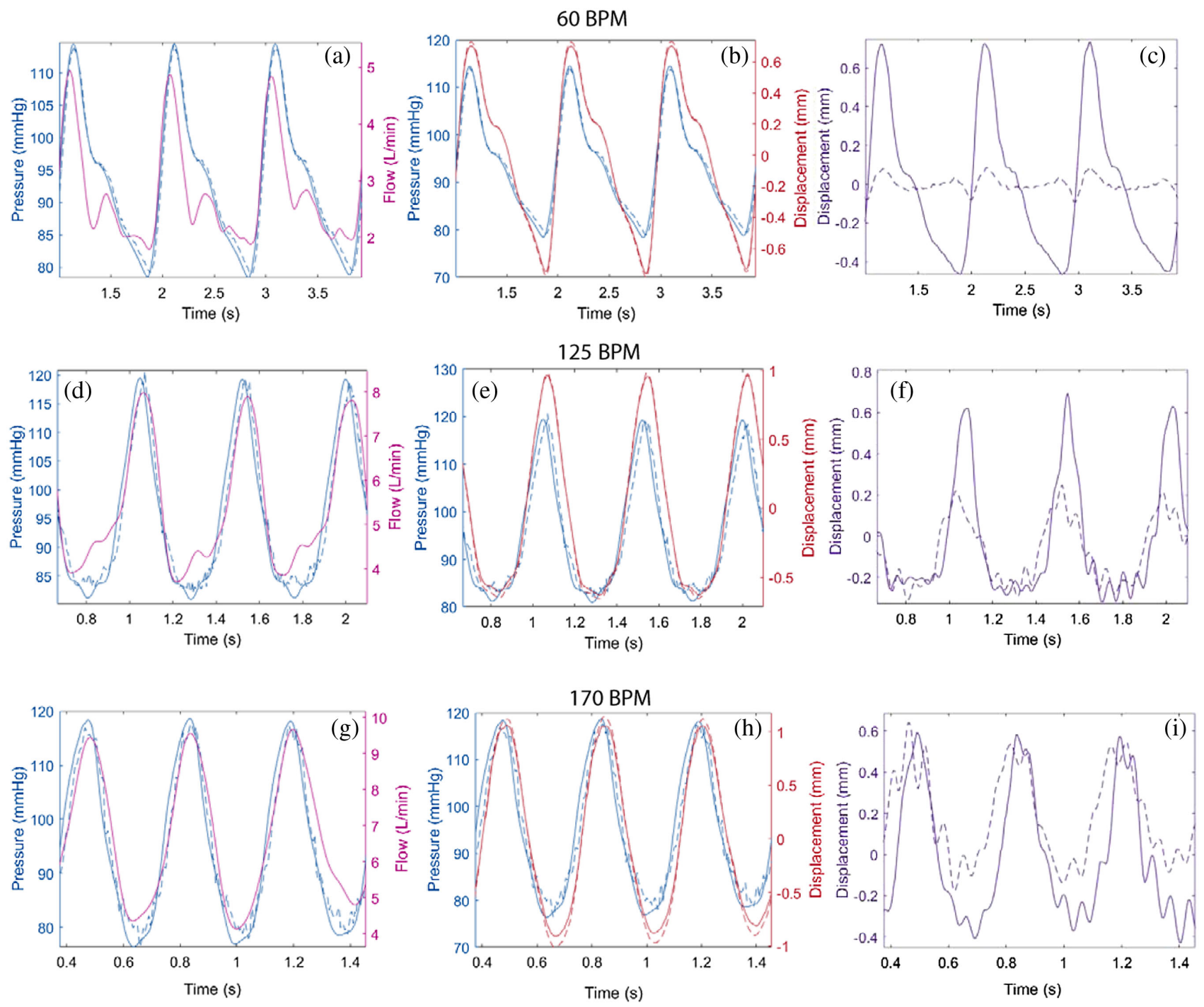

FIG. 3. Time responses of the aorta segment from donor XI at $60 \mathrm{bpm}$ : (a) fluid variables; (b) cyclic change of the aortic diameter and pressures; (c) cyclic bending oscillation of the aortic segment; at $125 \mathrm{bpm}$ : (d) fluid variables; (e) cyclic change of the aortic diameter and pressures; (f) cyclic bending oscillation of the aortic segment; at $170 \mathrm{bpm}$ : (g) fluid variables; (h) cyclic change of the aortic diameter and pressures; (i) cyclic bending oscillation of the aortic segment. Blue line, inlet pressure; blue dashed line outlet pressure; pink line outlet flowrate; red line change of the aortic diameter measured in vertical direction at mid-length of the aortic segment; red dashed line, change of the aortic diameter measured in horizontal direction; violet line bending amplitude at mid-length of the aortic segment measured in vertical direction; violet dashed line bending amplitude measured in horizontal direction. 
ensuring the dicrotic notch was created. The flow wave was anticipated with respect to the pressure wave, as reported in-vivo [8]. At $125 \mathrm{bpm}$, the flow and outlet pressure signals showed fewer higher harmonics than at $60 \mathrm{bpm}$. The shape of the wave was more sinusoidal, and the outlet pressure was slightly time delayed compared to the inlet pressure because of the pulse-wave propagation [32]. The highest pulse rate achieved during the experiments on the aortic segment from donor XI was $170 \mathrm{bpm}$. At this rate, the inlet and outlet pressure waves became significantly smoother with few superharmonic waves and visible pressure drop. There was also an increased delay between the outlet and the inlet pressure with respect to the test at $125 \mathrm{bpm}$.

The cyclic diameter change was plotted along with the inlet and outlet pressure to show that it rose with the pressure. However, during the descent, i.e., the contraction of the aorta, there was a delay of the displacement with respect to the pressure due to the viscoelasticity of the wall. The measured diameter change was almost identical in both the horizontal and vertical directions, which indicates a good symmetry of this aortic segment. At $60 \mathrm{bpm}$, the relative amplitude of the maximum cyclic change of the diameter, measured at the midlength of the specimen, was $9.5 \%$, which is consistent with in-vivo measurements for 40-year-old donors [7]. At an increased pulse rate of $125 \mathrm{bpm}$, the diameter changes exhibited fewer superharmonic components, but there was an increased time delay with respect to the inlet pressure. Nonetheless, the two waves had similar shapes. The response was again almost identical in horizontal and vertical directions, reaffirming that the symmetry is independent of the pulse rate and dependent on the specimen. However, the amplitude of the maximum relative diameter change was $10.5 \%$, slightly increasing from 60 to $125 \mathrm{bpm}$. At $170 \mathrm{bpm}$, the diameter change had a larger time delay with respect to the inlet pressure than in the previous two cases. Again, there was almost no discernible difference in the horizontal and vertical directions. The largest amplitude for the maximum cyclic diameter change was observed, attaining $13.1 \%$. This result is mainly due to the reduced mean pressure $(97.5 \mathrm{~mm} \mathrm{Hg})$ with respect to $125 \mathrm{bpm}(100 \mathrm{~mm} \mathrm{Hg})$ since the pulse pressure is almost the same in the two cases. A reduced mean pressure is associated with lower stiffness of the aorta since the material stiffens with strain due to the initial crimping of the collagen fibers. On the other hand, the difference in diameter change between 60 and $170 \mathrm{bpm}$ was mainly due to the different pulse pressure: $40 \mathrm{~mm} \mathrm{Hg}$ at $170 \mathrm{bpm}$ versus $36 \mathrm{~mm} \mathrm{Hg}$ at $60 \mathrm{bpm}$; in fact, the mean pressure had a $1 \%$ difference.

The bending oscillations were much larger in the vertical than in the horizontal direction because of the larger vertical deviation of the aortic segment from the straight tube geometry (see Table I). Higher frequency vibrations were seen in both directions at 125 and $170 \mathrm{bpm}$, which created a deviation of the bending response from the pressure wave. The peak-to-peak amplitude of the bending oscillation at $60 \mathrm{bpm}$ was $1.20 \mathrm{~mm}$ in the vertical direction and $0.18 \mathrm{~mm}$ in the horizontal direction. The total bending amplitude, as a result of the two directions, was comparable to the diameter change. At $125 \mathrm{bpm}$, the presence of higherfrequency vibrations increased significantly from $60 \mathrm{bpm}$ due to the excitation of the first natural mode of vibration of the clamped aortic segment. Again, the bending oscillations were larger in the vertical plane than the horizontal one, reaching amplitudes of $1.01 \mathrm{~mm}$ and $0.60 \mathrm{~mm}$, respectively. At $170 \mathrm{bpm}$, the bending oscillation amplitudes were more similar in the two directions, and there were even larger high-frequency vibrations. The amplitudes in the vertical and horizontal directions were $0.98 \mathrm{~mm}$ and $0.67 \mathrm{~mm}$.

Generally, cyclic diameter changes were similar in the two orthogonal directions, unless the aortic segment had significant stiffness asymmetry due to imperfections. Instead, bending oscillations had different amplitudes in the vertical and horizontal planes. These appeared simultaneously in the two planes due to initial deviation of the aortic segment from a straight tube. Therefore, the tested aortic segments were subjected to simultaneous cyclic diameter change and bending oscillations under pulsatile pressure and flow, as was shown for donor XI. Additional results for bending vibrations are presented in Appendix B.

\section{B. Frequency domain analysis}

The fast Fourier transform (FFT) of the bending oscillations in the vertical direction, for donor XI at $170 \mathrm{bpm}$, is presented in Fig. 4(a). It shows that a frequency peak arises around $16 \mathrm{~Hz}$. At all the pulse rates tested for donor XI, peaks were observed around this frequency. This observation suggests that the first natural bending mode of the clamped aortic segment, pressurized to $100 \mathrm{~mm} \mathrm{Hg}$ and filled with physiological saline solution, is around $16 \mathrm{~Hz}$. Figure 4(b) gives the frequency response function (FRF) between the bending oscillation and the inlet pressure for donor XI at $170 \mathrm{bpm}$. It clearly displays a single peak, in the frequency range of interest, corroborating the presence of the first bending natural mode of vibration around 16.1 Hz. This natural mode was excited by the pressure superharmonics, and it contributes to the dynamic bending response of the aortic segment with vibrations at its specific natural frequency.

The free length of the aortic segment was $118 \mathrm{~mm}$, and the initial prestretch was 1.15 times the initial length (refer to Table I). A prestretch value of 1.15 was chosen in accordance with reported experimental data based on the donor age, which was 40 years old in this case [28]. Small frequency variations were due to the slight differences while tuning the mean pressure of the flow at each pulse rate since the stiffness of the tissue increases with the strain. The pressure affected the level at which the tissue was preloaded. Therefore, the length and the prestretch played a significant role in the measured natural frequency. 

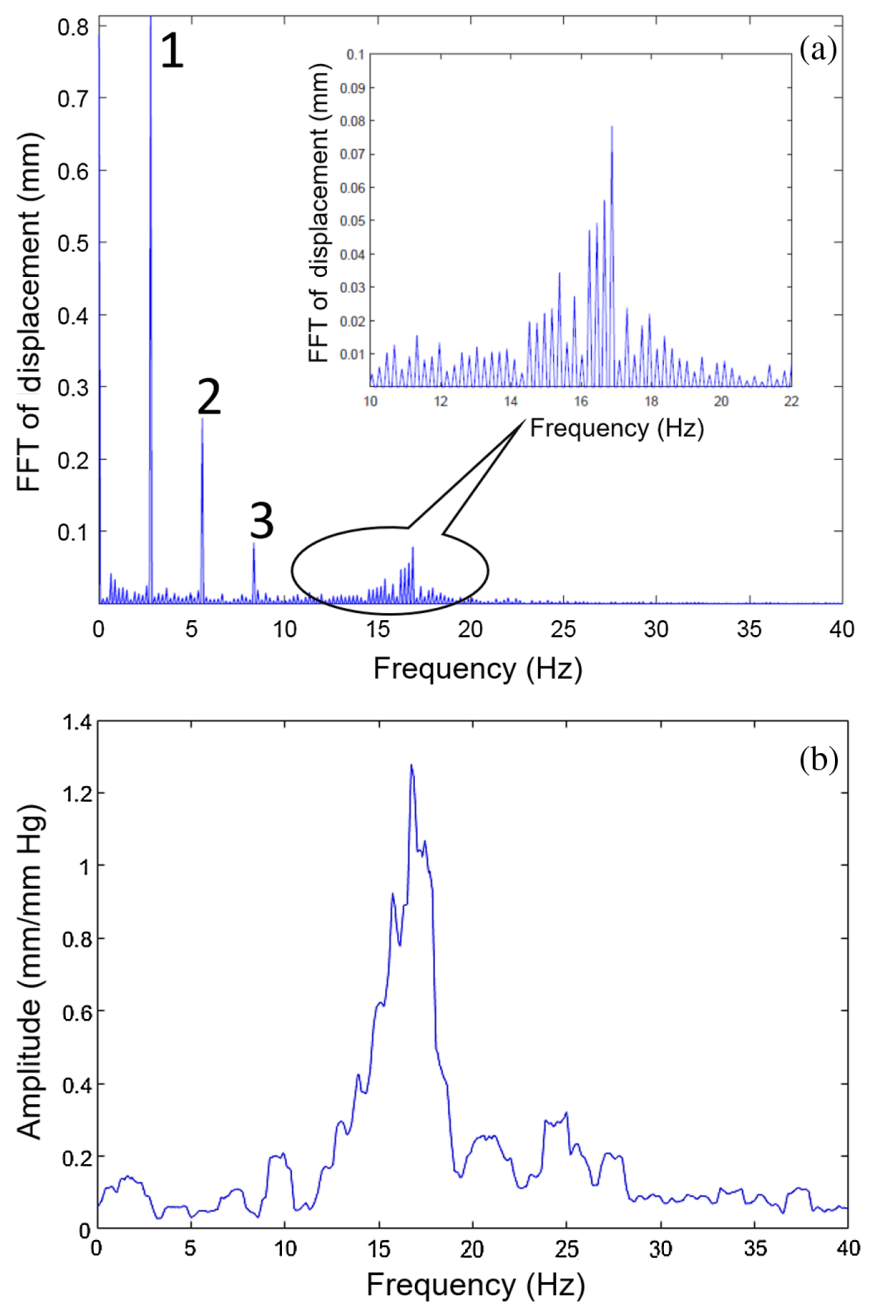

FIG. 4. Donor XI at $170 \mathrm{bpm}$ : (a) FFT of the bending oscillation in vertical direction where the numbers 1,2 and 3 correspond to the first, second and third harmonics, respectively; (b) FRF between the bending oscillation and the inlet pressure.

The same phenomenon was observed for all eleven aortic segments tested in the MCL. For all the cases, the first bending mode natural frequency is reported in the final row of Table I. The frequencies span a range of $10.1 \mathrm{~Hz}$, for donor XVIII, to $46.4 \mathrm{~Hz}$, for donor XVI, respectively. The smaller value was associated with the case with the smallest axial prestretch, while the highest natural frequency occurred for the aorta with the shortest length and the largest prestretch. This case suggests that the natural frequency of the first bending mode is dependent upon the intrinsic material properties of the specimen, as well as the geometry and testing parameters. When observing the bending oscillations for the two most extreme cases, large contributions of the first natural mode of vibration were seen, albeit smaller for donor XVIII than donor XVI. The bending response was composed of a sinusoidal wave superimposed with higher-frequency oscillations. These were created by the free vibration of the first bending mode, which was excited by the superharmonics of the cyclic pulsation. The pressure generated dynamic axial stresses on the aortic segment that was initially bent due to the deviation of its axis.

\section{VISCOELASTIC PROPERTIES}

\section{A. Loss factor and storage modulus identification}

The hysteresis loops of the dynamic diameter change were used to obtain the loss factors, $\eta$. Figure 5(a) shows the plot of the hysteresis loop for the aortic segment from donor IX at $63 \mathrm{bpm}$ that highlights the areas of interest for calculating the loss factor.

For a complete vibration cycle, the energy dissipated by an aorta subjected to pulsatile pressure is [17]

$$
\Delta W_{d}=\int_{\text {cycle }} p A d x
$$

where $p$ is the pulsatile pressure, $A$ is the reference area where the pressure is applied, and $x$ is the dynamic radial displacement of the aorta wall (half of the diameter change) measured at the midlength of the specimen. Equation (3) describes the area contained within the hysteresis loop for the pressure versus radius change. This equation gives the relationship between the area inside the hysteresis loop and the dissipated energy as

$$
A_{\text {inside loop }}=2 \Delta W_{d} / A \text {. }
$$

The coefficient 2 in Eq. (4) arises from the fact that the hysteresis loop represents the pressure variation versus diameter change. The hysteresis loop, shown in Fig. 5(a), can be divided into two curves: the top half refers to pressurization and the lower to depressurization. They join at the two extrema of the horizontal direction of the loop, $x_{\min }$ and $x_{\max }$.

The elastic relationship describing the pressure-diameter change of the viscoelastic aorta, under pulsatile pressure, was assumed to be the average of the upper half and lower half of the loop; it is the backbone curve of the loop drawn in red in Fig. 5(a). The storage energy $W_{S}$ for one-quarter of the cycle [17] was given by

$$
W_{S}=\frac{\frac{1}{2} S_{1}+\frac{1}{2} S_{2}}{2} A,
$$

where $S_{1}$ and $S_{2}$ are the areas of the two curvilinear triangles shown in Fig. 5(a). Then, the loss factor $\eta$ was obtained as [17]

$$
\eta=\frac{\Delta W_{d}}{2 \pi W_{s}}=\frac{A_{\text {inside loop }}}{\pi\left(S_{1}+S_{2}\right)} .
$$

This expression refers to the parameters described in Fig. 5(a). Since Eq. (6) is a ratio of two areas, it carries no measurement units. The loss factor coincides with the loss tangent in the case of linear viscoelasticity. 

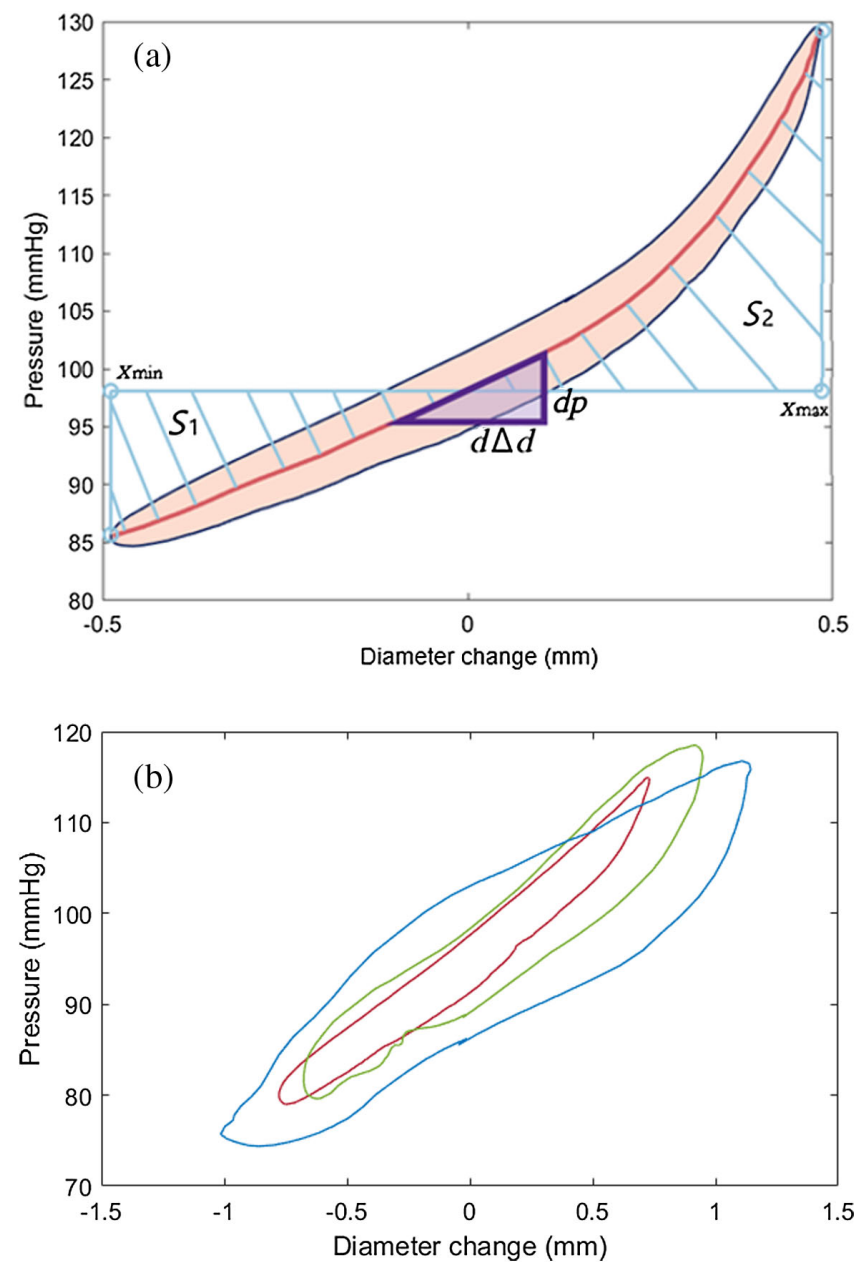

FIG. 5. Hysteresis loop of the pressure-diameter change: (a) donor IX at $63 \mathrm{bpm}$ in the vertical direction at mid-length of the aortic segment. Area inside the loop is proportional to the energy loss. The average of areas $S_{1}$ and $S_{2}$, found using the middle line of the loop (red line) and the horizontal line passing through it at $x=0$, is proportional to the storage energy. The triangle (violet line) describes the storage modulus computed as the slope at the origin $(d p / d \Delta d)$; (b) donor XI hysteresis loop obtained from the cyclic change of aortic diameter and inlet pressure measured in horizontal direction:, red line $60 \mathrm{bpm}$; green line $100 \mathrm{bpm}$; blue line, $170 \mathrm{bpm}$.

The storage modulus of the aorta, $E^{\prime}$, is defined as a function of the slope $d p / d \Delta d$ at the origin of the backbone curve of the hysteresis loop

$$
E^{\prime}=\frac{3 R_{i}^{2} R_{e}}{2 h R_{m}} \frac{d p}{d \Delta d}
$$

where $p$ is the internal pressure, $\Delta d$ is the diameter change of the aorta, $R_{i}$ is the inner radius of the aorta, $R_{e}$ is the external radius of the aorta, $h$ is the thickness of the wall of the aorta, and $R_{m}$ is the mean radius of the aortic wall. This slope is the hypotenuse of the purple triangle for the pressure versus external diameter change, shown in
Fig. 5(a). A complete derivation of Eq. (7) is included in Appendix C, assuming an incompressible, isotropic, homogeneous, and elastic hollow cylinder.

The hysteresis loops for donor XI in the horizontal direction are summarized in Fig. 5(b), for pulse rates of 60, 100 , and $170 \mathrm{bpm}$. The pressures for the loops ranged from 75 to $120 \mathrm{~mm} \mathrm{Hg}$, and the maximum cyclic diameter change was $2.09 \mathrm{~mm}$. The cyclic diameter change was almost identical in the vertical and horizontal planes. The area inside the loop increases with the pulse rate, which indicates that more energy is dissipated during each pulsation cycle at higher frequency.

For donor XI, the loss factor was 0.09 at $20 \mathrm{bpm}$ and 0.20 at $60 \mathrm{bpm}$, and it increased to 0.42 and 0.51 at $125 \mathrm{bpm}$ and $170 \mathrm{bpm}$, respectively. While an increase of the material loss factor was expected with the frequency increase from 20 to $60 \mathrm{bpm}$, any further increase with higher frequency was found to be modest in the literature [9,25]. Uniaxial harmonic loading experiments were performed on axial and circumferential strips of aortic tissue, after the MCL test. These results are presented in Appendix D and are close enough to those in the MCL at $60 \mathrm{bpm}$. Therefore, this large increase of the loss factor is attributed to the interaction between the pulsatile flow and the aorta wall. The fluidstructure interaction largely amplifies the dissipation with the increase of the pulse rate and flowrate.

While most of the data and figures presented in the previous sections pertain to donor XI, the values of the diameter change, the loss factors, and the storage moduli are reported for all eleven donors in Appendix A.

At some pulse rates for certain aortic segments, the hysteresis loops intersected themselves or were too noisy to accurately extract values for the loss factor. This phenomenon was more common for bending oscillations due to the superimposed vibrations. Therefore, these values were omitted from the data to avoid misleading results. The typical error for the loss factor was \pm 0.01 . This value was estimated by using different periods of the time response to evaluate $\eta$ since measured periods were similar but not identical. Similarly, the estimated error for the storage modulus computation was about $5 \%$. This value varied according the selected period and the order of the fitting polynomial to find the slope at the origin.

\section{B. Variation of viscoelastic properties with age}

To interpret the significance of the results in biomedical engineering applications, the relationships between the diameter changes, loss factors, and storage moduli with age were studied. The correlation between age and the three parameters at $60 \mathrm{bpm}$ is given in Fig. 6. The maximum cyclic diameter change is shown to be inversely correlated with the age of the donor. As the donors age, the diameter exhibits smaller cyclic amplitude changes with each pulsation. This result is consistent with in-vivo $\mathrm{CT}$ imaging at rest of 14 individuals equally divided into two age groups, 

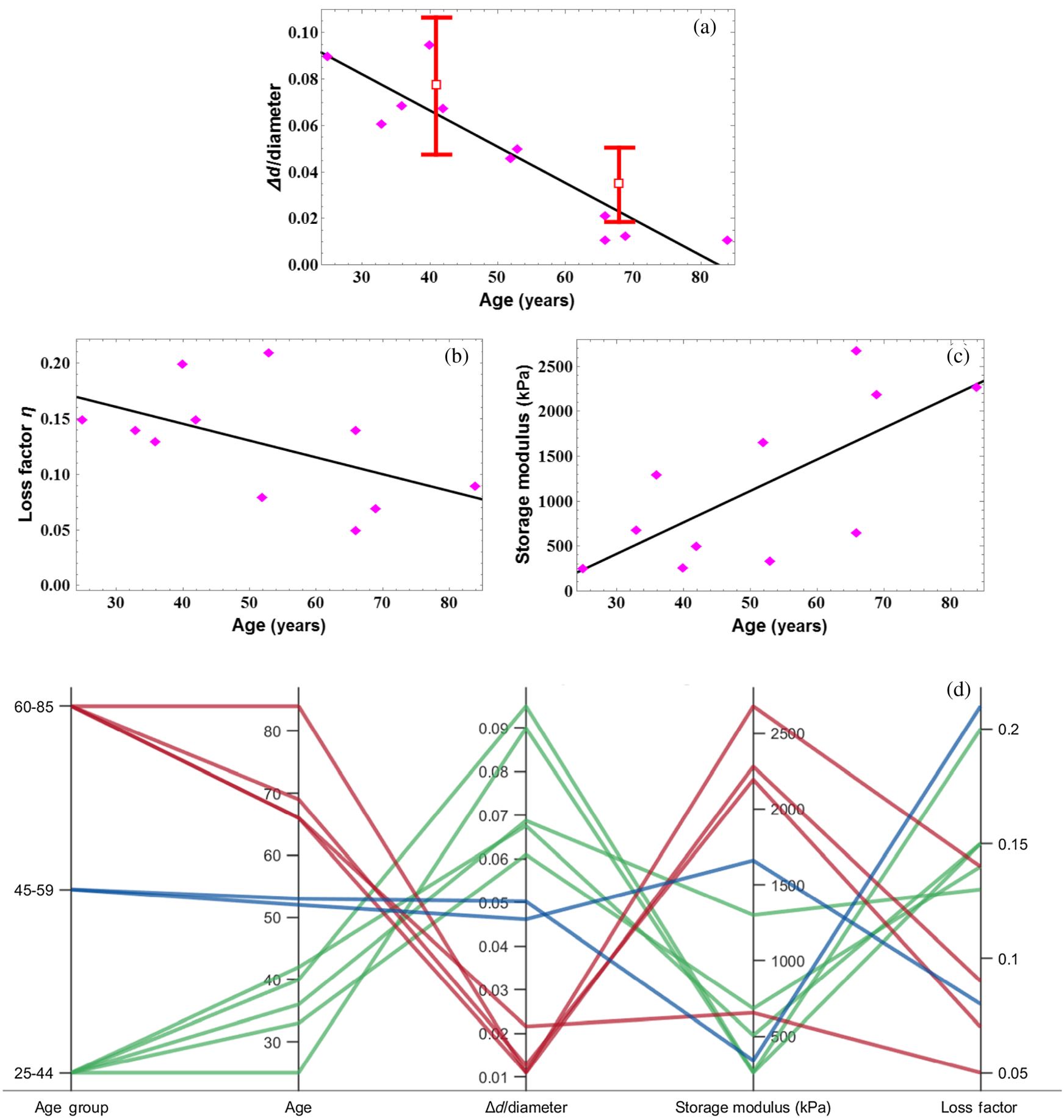

FIG. 6. Correlation for cyclic diameter change and viscoelastic parameters with age at $60 \mathrm{bpm}$, for the eleven donors: (a) maximum diameter change at $60 \mathrm{bpm}$ pulse rate normalized with respect to the diameter $(\Delta d /$ diameter, experimental points diamond) with linear regression $(0.12889-0.00156 \mathrm{~A}, \mathrm{~A}=$ age (years); $\mathrm{p}$-value $=0.000013$; Pearson's $\mathrm{R}=-0.912$ ). Data obtained as the average of diameter changes at the proximal and distal extremities of thoracic descending aortas from in vivo CT images from literature is reported for comparison [7]; average values and standard deviations for two age groups (41 \pm 7 years; $68 \pm 6$ years) of seven individuals each are given (two red error bars); (b) loss factor with linear regression $(0.20602-0.00151 \mathrm{~A}, \mathrm{~A}=$ age (years); p-value $=0.0915$; Pearson's $\mathrm{R}=-0.535)$; (c) storage modulus with linear regression $(-636.26+35.024 A, \mathrm{~A}=$ age (years); p-value $=0.0129$; Pearson's $\mathrm{R}=0.706$ ); (d) parallel coordinate plot demonstrating the relationship between the relative diameter change $\Delta d /$ diameter, storage modulus and loss factor with age, for 3 age groups: green line, 25-44 years old; blue line, 45-59 years old; red line, 60-85 years old. 
34-48 and 62-74 years old, comparing the average diameter change with age [7]. This comparison is shown in Fig. 6(a) by red bars delimiting the boundaries (standard deviation) of the diameter expansion for the two age groups. The experimental data obtained fall within the same bounds, confirming that the MCL is capable of reproducing in-vivo conditions. The decrement of the diameter expansion with age gives a reduced Windkessel effect in the elderly.

The dependence between age and the viscoelastic material properties was evaluated for all the donors. The loss factor was shown to decrease with age, Fig. 6(b), whereas the storage modulus increased, Fig. 6(c). At $60 \mathrm{bpm}$, the loss factors ranged from 0.05 to 0.21 , and the storage moduli from 259 to $2680 \mathrm{kPa}$. At $60 \mathrm{bpm}$, the loss factor can be mainly attributed to the dissipation by the aorta wall, while the fluid-structure interaction contribution dominated at larger heart rates. The increase of the loss factor with the pulse rate was not observed in tests performed on Dacron aortic grafts, using both saline solution and a glycerine/water mixture, since Dacron grafts are significantly stiffer in the radial direction [5]. Therefore, the loss factor seems to show a dependency from the radial expansion of the aorta. These findings are confirmed by the tests with a water/glycerin mixture, the blood analogue fluid, on two aortas (donors XIX and XXI). They show an almost identical loss factor obtained with the analogue mixture and the saline solution at $60 \mathrm{bpm}$, confirming a small fluid contribution. The loss factor slowly increased with the pulse rate for the mixture with respect to the one for saline solution, but the difference was practically negligible up to $120 \mathrm{bpm}$. Then, the loss factor progressively increases up to $24 \%$ at $176 \mathrm{bpm}$. The detailed comparison between tests with saline solution and the water/glycerin mixture is given in Appendix A. It was observed that the material internal damping capacity decreased with age. The trend between age and storage modulus corroborated the finding of smaller diameter variation with age [7]. The higher storage modulus for older donors reflected the development of pathology causing aortic tissue to stiffen, like atherosclerotic plaque and calcification. Increased aortic stiffness has a major effect on pulse pressure (the difference between systolic and diastolic blood pressure), wave reflections, and, above all, cardiovascular risk [33]. The eleven donors were divided into three age groups in Fig. 6(d): 25-44, 45-59, and 60-85 years old. The three parameters and age are linked by lines for each aortic segment at $60 \mathrm{bpm}$; different age groups have lines of different color.

\section{Effect of pulse rate on the viscoelasticity}

The effect of the pulse rate on the viscoelastic parameters was also evaluated. The variation of relative diameter change, the loss factor, and the storage modulus with the pulse rate is shown in Figs. 7(a)-7(c), respectively.
The values are grouped according to the three aforementioned age ranges. A modest increase in the diameter change (normalized with respect to the external central diameter at $30 \mathrm{~mm} \mathrm{Hg}$ ) is observed in Fig. 7(a) for the two highest pulse rates, 145 and $170 \mathrm{bpm}$, for all three age groups, showing that dynamics plays a role. From Fig. 7(b), it is possible to see that the loss factor strongly depends on the pulse rate. As the pulse rate increases from $60 \mathrm{bpm}$ to $170 \mathrm{bpm}$, the loss factor values more than double.
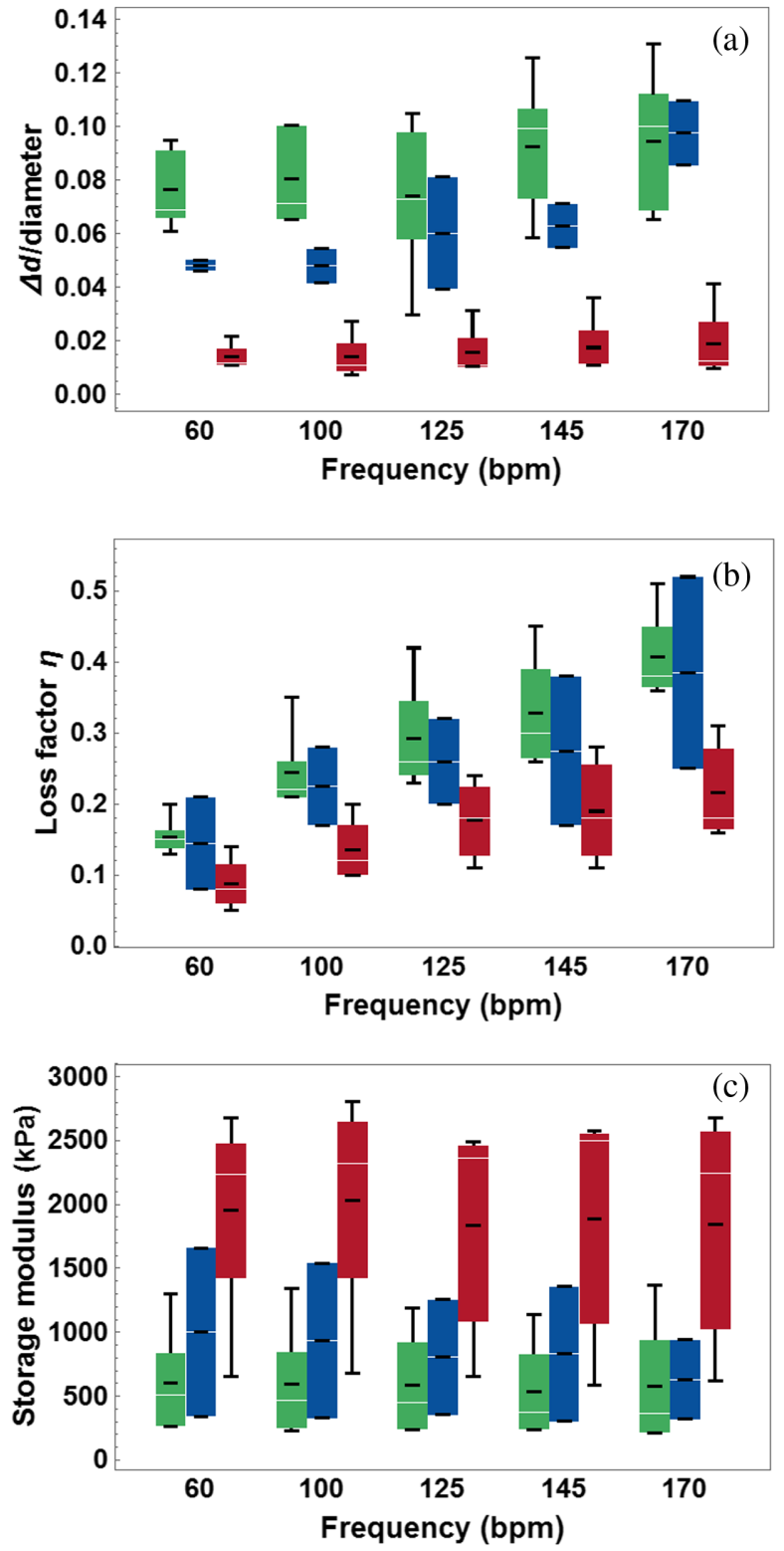

FIG. 7. Statistical analysis of the viscoelastic parameters at different pulse rates; maximum, minimum, 1st quartile, 3rd quartile, average (black horizontal line inside the box) and median (white horizonal line in the box) are reported for 3 age groups, green rectangular box, 25-44 years old; blue rectangular box, 45-59 years old; red rectangular box, 60-85 years old: (a) maximum relative diameter change; (b) loss factor; (c) storage modulus. 
This trend impacts the younger donors (green and blue groups) more than the elder one (red group). At higher pulse rates, the contribution of fluid-structure interaction on the loss factor is larger, and the reduced diameter change during pulsation seems to be the cause of the reduced dissipative effect of the flow for the elder group.

On the other hand, the pulse rate has little impact on the storage modulus. As observed in Fig. 7(c), there is essentially no variation of the storage modulus with increasing pulse rate. Consistent with Fig. 6, the storage modulus is dependent upon the age of the donors: The younger the donor, the smaller the storage modulus. Therefore, the stiffness of the aortas is intrinsic to the tissue, as the effect of the fluid-structure interaction is negligible in the physiological regime. Also, published data indicate that there would be little variation of the storage modulus with the pulse rate within the physiological range $[9,25]$. Instead, a significant change of the storage modulus occurs from static to dynamic conditions. This result reaffirms the importance of viscoelastic material characterization for the aorta, rather than a purely static one.

\section{Comparison with Dacron aortic grafts}

A comparison of the mechanical properties of human descending thoracic aortas to current commercial Dacron aortic grafts was performed. Dacron grafts are widely used for aortic repairs. A Hemashield Platinum woven double velour straight graft, made by Maquet (model 175428P) for descending thoracic aorta replacement with a nominal diameter of $28 \mathrm{~mm}$, was tested in Ref. [5] at different pulse rates by using a MCL (see Ref. [5] for details of the graft geometry, material properties, and experimentation). The axial prestretch was 1.3. A Hemashield Platinum woven double velour fourbranch graft, made by Maquet (model 175928P), was tested by the authors with axial prestretch 1.33 (the initial length was $96.5 \mathrm{~mm}$, and the stretched length was $128.5 \mathrm{~mm}$; the straight portion without branches was tested).

Figure 8 presents the variation of relative diameter change with the pulse rate for the 60-85-year-old group of aorta donors, which are the stiffest ones in the present study, versus the two similar Dacron aortic grafts subjected to different axial prestretch. The results show a major mismatch between the cyclic expansion of the human aorta for elder humans versus commercial Dacron grafts. This discrepancy is largely magnified for younger age groups, as shown by comparing Figs. 7(a) and 8.

The storage modulus in the circumferential direction of the Dacron graft, which was identified in Ref. [5] from a uniaxial tensile test, was reported to be between 140 and $250 \mathrm{MPa}$ in the frequency range of interest. The values were influenced by the amount of prestretch. Tests on the branched graft by the present authors gave storage modulus values from 108 to $125 \mathrm{MPa}$; they were obtained from tests in the MCL with a glycerine/water mixture. Figure 7(c) shows that the storage modulus of the tested human aortas was always below $3 \mathrm{MPa}$. This fact clarifies that Dacron grafts are much stiffer in circumferential direction as compared to human aortas.

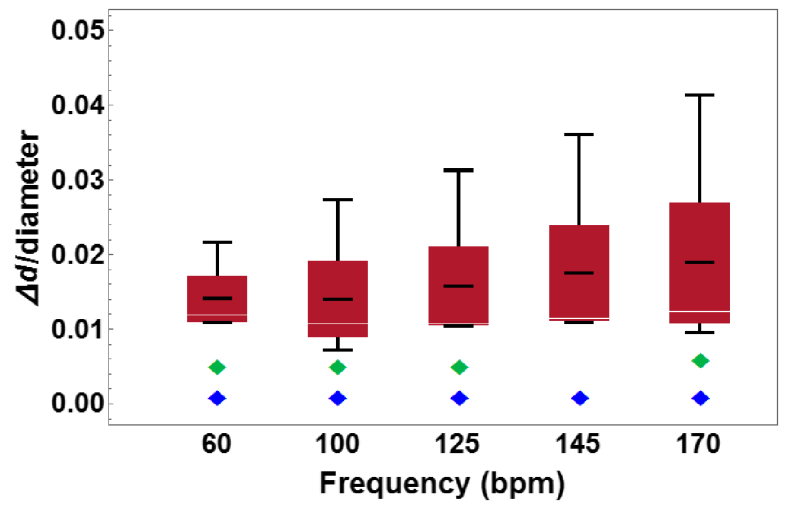

FIG. 8. Comparison of the maximum relative diameter change for human aortas within the 60-85 years age group and woven Dacron aortic grafts at different pulse rates. red box, statistical analysis of the data for human aortas for the 60-85 years group; green diamond, Dacron graft tested in [5] with an axial pre-stretch of 1.3; blue diamond, Dacron graft tested by the present authors with an axial pre-stretch of 1.33 .

Therefore, Dacron grafts are unable to provide any Windkessel effect. In addition, they alter the nonlinear dynamics of the aorta and the blood flow.

\section{CONCLUSIONS AND OUTLOOK}

The aims of the experiment were successfully met: The viscoelastic material parameters for the human descending thoracic aorta were identified by using a MCL specifically conceived to reproduce physiological pulsatile flow. The loss factors reported were representative of both the aorta and the flow. They would be difficult to measure in-vivo at higher pulse rates. Furthermore, the local pressure measurement would be invasive. This result presses on the importance of ex-vivo studies. However, the measurement ex-vivo requires a perfectly tuned MCL since the tissue alone is insufficient to obtain the necessary information. The MCL developed for this study was able to reproduce an accurate physiological flow mimicking pressure, pulse rate, and temperature conditions. Viscoelastic material characterizations of human descending thoracic aortas using a MCL had never been explored until now. In the present case, state-of-the-art equipment (including four laser Doppler vibrometers) was utilized.

The dynamics and the material properties of the aorta obtained from these experiments are essential for advancing the field of cardiovascular medical device design. We envision that physicians and biomedical engineers can collaborate to create aortic grafts in advanced materials that effectively reproduce the natural behavior of the human aorta by using the data identified in this study.

\section{ACKNOWLEDGMENTS}

The authors acknowledge the financial support of the NSERC Discovery Grant and the Canada Foundation for 
Innovation John R. Evans Leaders Fund. Dr. Goffredo Arena, M. D., arranged the collaboration with Transplant Québec that provided the aortas, and helped us with the specimen preparation. Ali Kassab prepared the aorta samples. Dr. Meisam Asgari performed histological analyses and obtained the SHG image. The authors thank Professor Rosaire Mongrain who generously supplied the Harvard Apparatus pump that was modified for the experiments.

\section{APPENDIX A: DIAMETER CHANGE, LOSS FACTOR, AND STORAGE MODULUS DATA}

Experimental data of pressure waves, change in diameter, bending displacements, and corresponding loss factors and storage moduli for each of the eleven donors are summarized in Table II. The displacement measurement

TABLE II. Experimental data of the pressure, diameter and bending displacements, loss factors, and storage moduli for the eleven aortic segments tested in the mock circulatory loop. The loss factor $\eta$ associated with unsmooth and self-intersecting hysteresis loops were not reported (the corresponding value is indicated as n.a. for "not available").

\begin{tabular}{lccccc}
\hline \hline Donor VI & & & & & \\
\hline Pulse rate $(\mathrm{bpm})$ & 56 & 98 & 127 & 145 & 172 \\
\hline Mean pressure $(\mathrm{mmHg})$ & 95.8 & 93.9 & 107.4 & 97.0 & 96.6 \\
Pulse pressure $(\mathrm{mmHg})$ & 58.7 & 46.1 & 41.1 & 39.7 & 44.5 \\
Max $\Delta d(\mathrm{~mm})$ & 1.25 & 1.32 & 0.55 & 1.08 & 1.29 \\
Max $\Delta b(\mathrm{~mm})$ & 2.39 & 1.96 & 1.62 & 1.85 & 2.01 \\
$\eta$ diameter change & 0.15 & 0.22 & 0.23 & 0.27 & 0.39 \\
Storage modulus $(\mathrm{kPa})$ & 507 & 468 & 651 & 509 & 506 \\
$\eta$ bending & 0.21 & 0.28 & 0.29 & 0.31 & 0.39 \\
\hline \hline
\end{tabular}

Donor VII

\begin{tabular}{lcccccc}
\hline Pulse rate $(\mathrm{bpm})$ & 34 & 55 & 97 & 130 & 145 & 170 \\
\hline Mean pressure $(\mathrm{mmHg})$ & 103 & 109.8 & 110.7 & 104.1 & 96.5 & 101.7 \\
Pulse pressure $(\mathrm{mmHg})$ & 31.9 & 28.0 & 34.8 & 34.1 & 29.9 & 35.3 \\
Max $\Delta d(\mathrm{~mm})$ & 0.68 & 0.49 & 0.62 & 0.71 & 0.82 & 0.94 \\
Max $\Delta b(\mathrm{~mm})$ & 0.58 & 0.49 & 0.65 & 0.56 & 0.79 & 0.65 \\
$\eta$ diameter change & 0.05 & 0.05 & 0.10 & 0.11 & 0.11 & 0.16 \\
Storage modulus $(\mathrm{kPa})$ & 642 & 655 & 679 & 654 & 589 & 620 \\
$\eta$ bending & 0.10 & 0.08 & 0.15 & 0.18 & 0.20 & 0.28
\end{tabular}

Donor VIII

\begin{tabular}{lccccc}
\hline Pulse rate $(\mathrm{bpm})$ & 58 & 103 & 125 & 137 & 162 \\
\hline Mean pressure $(\mathrm{mmHg})$ & 104.0 & 100.5 & 91.6 & 100.3 & 95.5 \\
Pulse pressure $(\mathrm{mmHg})$ & 42.2 & 41.8 & 39.7 & 36.0 & 38.7 \\
Max $\Delta d(\mathrm{~mm})$ & 0.83 & 0.98 & 1.46 & 1.28 & 1.97 \\
Max $\Delta b(\mathrm{~mm})$ & 0.29 & 0.31 & 0.36 & 0.38 & 0.53 \\
$\eta$ diameter change & 0.08 & 0.17 & 0.20 & 0.17 & 0.25 \\
Storage modulus $(\mathrm{kPa})$ & 1661 & 1542 & 1257 & 1359 & 942 \\
$\eta$ bending & 0.08 & n.a. & 0.22 & 0.33 & 0.37 \\
\hline \hline
\end{tabular}

TABLE II. (Continued)

Donor IX

\begin{tabular}{lllllllll}
\hline Pulse rate (bpm) & 20.4 & 63 & 86 & 103 & 126 & 142 & 160 & 172
\end{tabular}

Mean pressure $\quad 100.8100 .296 .999 .498 .496 .999 .694 .1$ (mmHg)

$\begin{array}{lllllllll}\text { Pulse pressure } \quad 35.4 & 45.5 & 36.0 & 34.4 & 33.8 & 34.8 & 36.7 & 42.4\end{array}$ (mmHg)

$\begin{array}{llllllllll}\operatorname{Max} \Delta d(\mathrm{~mm}) & 1.22 & 1.11 & 1.23 & 0.92 & 0.87 & 1.21 & 1.18 & 1.89\end{array}$

$\begin{array}{llllllllll}\operatorname{Max} \Delta b(\mathrm{~mm}) & 0.15 & 0.22 & 0.15 & 0.12 & 0.14 & 0.21 & 0.19 & 0.30\end{array}$

$\begin{array}{llllllllll}\eta \text { diameter change } & 0.09 & 0.21 & 0.24 & 0.28 & 0.32 & 0.38 & 0.43 & 0.52\end{array}$

$\begin{array}{lllllllll}\text { Storage modulus } & 271 & 340 & 311 & 328 & 354 & 303 & 320 & 321\end{array}$ $(\mathrm{kPa})$

$\eta$ bending $\quad 0.090 .18$ n.a. n.a. n.a. n.a. n.a. n.a.

Donor XI

\begin{tabular}{|c|c|c|c|c|c|c|c|}
\hline Pulse rate (bpm) & 19.3 & 60 & 80 & 100 & 125 & 150 & 170 \\
\hline $\begin{array}{l}\text { Mean pressure } \\
(\mathrm{mmHg})\end{array}$ & 102.1 & 96.5 & 96.2 & 102.1 & 100.0 & 99.2 & 97.5 \\
\hline $\begin{array}{l}\text { Pulse pressure } \\
\quad(\mathrm{mmHg})\end{array}$ & 39.6 & 36.0 & 39.1 & 38.7 & 39.8 & 40.8 & 40.0 \\
\hline $\operatorname{Max} \Delta d(\mathrm{~mm})$ & 1.99 & 1.52 & 1.74 & 1.60 & 1.68 & 2.01 & 2.09 \\
\hline $\operatorname{Max} \Delta b(\mathrm{~mm})$ & 0.70 & 1.21 & 1.07 & 1.14 & 1.17 & 1.21 & 1.19 \\
\hline$\eta$ diameter change & 0.09 & 0.20 & 0.26 & 0.35 & 0.42 & 0.45 & 0.51 \\
\hline $\begin{array}{l}\text { Storage Modulus } \\
\quad(\mathrm{kPa})\end{array}$ & 321 & 264 & 260 & 232 & 234 & 239 & 226 \\
\hline$\eta$ bending & 0.08 & 0.19 & 0.25 & n.a. & n.a. & n.a. & n.a. \\
\hline
\end{tabular}

\begin{tabular}{|c|c|c|c|c|c|}
\hline \multicolumn{6}{|l|}{ Donor XV } \\
\hline Pulse rate (bpm) & 66 & 100 & 125 & 150 & 180 \\
\hline $\begin{array}{l}\text { Mean pressure } \\
(\mathrm{mmHg})\end{array}$ & 97.0 & 100.1 & 101.7 & 106.5 & 105.5 \\
\hline $\begin{array}{l}\text { Pulse pressure } \\
(\mathrm{mmHg})\end{array}$ & 49.1 & 34.3 & 44.9 & 52.8 & 57.3 \\
\hline $\operatorname{Max} \Delta d(\mathrm{~mm})$ & 0.28 & 0.16 & 0.23 & 0.25 & 0.28 \\
\hline $\operatorname{Max} \Delta b(\mathrm{~mm})$ & 0.50 & 0.31 & 0.41 & 0.59 & 0.46 \\
\hline$\eta$ diameter change & 0.07 & 0.10 & n.a. & n.a. & n.a. \\
\hline $\begin{array}{l}\text { Storage modulus } \\
\quad(\mathrm{kPa})\end{array}$ & 2195 & 2161 & n.a. & n.a. & n.a. \\
\hline$\eta$ bending & 0.10 & 0.15 & n.a. & n.a. & n.a. \\
\hline \multicolumn{6}{|l|}{ Donor XVI } \\
\hline Pulse rate (bpm) & 60 & 100 & 125 & 155 & 180 \\
\hline $\begin{array}{l}\text { Mean pressure } \\
(\mathrm{mmHg})\end{array}$ & 101.8 & 100.2 & 96.8 & 94.7 & 98.2 \\
\hline $\begin{array}{l}\text { Pulse pressure } \\
\quad(\mathrm{mmHg})\end{array}$ & 43.5 & 40.4 & 42.3 & 46.7 & 44.9 \\
\hline $\operatorname{Max} \Delta d(\mathrm{~mm})$ & 1.06 & 1.14 & 1.17 & 1.73 & 1.74 \\
\hline $\operatorname{Max} \Delta b(\mathrm{~mm})$ & 0.07 & 0.10 & 0.17 & 0.33 & 0.41 \\
\hline$\eta$ diameter change & 0.14 & 0.21 & n.a. & n.a. & n.a. \\
\hline $\begin{array}{l}\text { Storage modulus } \\
\quad(\mathrm{kPa})\end{array}$ & 683 & 675 & n.a. & n.a. & n.a. \\
\hline$\eta$ bending & n.a. & n.a. & n.a. & n.a. & n.a. \\
\hline
\end{tabular}


TABLE II. (Continued)

\begin{tabular}{lccccc}
\hline Donor XVII & & & & & \\
\hline Pulse rate $(\mathrm{bpm})$ & 62 & 100 & 128 & 150 & 174 \\
\hline Mean pressure $(\mathrm{mmHg})$ & 96.7 & 100.1 & 98.5 & 97.6 & 105.1 \\
Pulse pressure $(\mathrm{mmHg})$ & 33.0 & 35.7 & 36.1 & 37.7 & 38.4 \\
Max $\Delta d(\mathrm{~mm})$ & 1.32 & 1.25 & 1.4 & 1.5 & 1.25 \\
Max $\Delta b(\mathrm{~mm})$ & 4.37 & 4.88 & 4.85 & 6.30 & 5.35 \\
$\eta$ diameter change & 0.13 & 0.23 & 0.27 & 0.33 & 0.36 \\
Storage modulus $(\mathrm{kPa})$ & 1300 & 1345 & 1193 & 1139 & 1367 \\
$\eta$ bending & 0.14 & 0.25 & 0.31 & 0.38 & 0.44
\end{tabular}

\begin{tabular}{lccccc}
\hline \hline Donor XVIII & & & & & \\
\hline Pulse rate (bpm) & 66 & 103 & 126 & 145 & 174 \\
\hline Mean pressure (mmHg) & 99.4 & 101.0 & 99.8 & 102.9 & 104.7 \\
Pulse pressure (mmHg) & 40.1 & 42.3 & 42.7 & 44.3 & 42.5 \\
Max $\Delta d(\mathrm{~mm})$ & 0.23 & 0.22 & 0.22 & 0.23 & 0.20 \\
Max $\Delta b(\mathrm{~mm})$ & 0.12 & 0.14 & 0.15 & 0.11 & 0.10 \\
$\eta$ diameter change & 0.09 & 0.14 & 0.17 & 0.18 & n.a. \\
Storage modulus (kPa) & 2281 & 2481 & 2360 & 2498 & 2677 \\
$\eta$ bending & 0.08 & 0.13 & 0.17 & 0.17 & n.a. \\
\hline \hline
\end{tabular}

Donor XIX (saline solution)

\begin{tabular}{lcccccc}
\hline Pulse rate (bpm) & 60 & 95 & 118 & 154 & 164 & 183 \\
\hline Mean pressure (mmHg) & 99.8 & 98.2 & 96.5 & 98.1 & 98.0 & 100.5 \\
Pulse pressure (mmHg) & 37.1 & 39.8 & 39.5 & 38.1 & 36.3 & 38.2 \\
Max $\Delta d(\mathrm{~mm})$ & 0.20 & 0.20 & 0.20 & 0.21 & 0.22 & 0.22 \\
Max $\Delta b(\mathrm{~mm})$ & 0.05 & 0.08 & 0.1 & 0.11 & 0.09 & 0.06 \\
$\eta$ diameter change & 0.14 & 0.20 & 0.24 & 0.28 & 0.31 & 0.37 \\
Storage modulus (kPa) & 2680 & 2808 & 2491 & 2572 & 2243 & 2015 \\
$\eta$ bending & 0.1 & n.a. & n.a. & 0.29 & n.a. & 0.39 \\
\hline \hline & & & & & & \\
\hline Donor XIX (water/glycerin mix) & & & & \\
\hline Pulse rate (bpm) & 64 & 108 & 127 & 152 & 164 & 186 \\
\hline Mean pressure (mmHg) & 97.6 & 100.3 & 96.7 & 100.6 & 101.1 & 101.2 \\
Pulse pressure (mmHg) & 39.8 & 37.2 & 39.2 & 35.6 & 35.9 & 38.6 \\
Max $\Delta d$ (mm) & 0.25 & 0.23 & 0.23 & 0.22 & 0.24 & 0.26 \\
Max $\Delta b$ (mm) & 0.08 & 0.09 & 0.08 & 0.08 & 0.07 & 0.09 \\
$\eta$ diameter change & 0.15 & 0.22 & 0.24 & 0.30 & 0.38 & 0.45 \\
Storage modulus (kPa) & 2112 & 2186 & 2342 & 2310 & 1915 & 2003 \\
$\eta$ bending & 0.14 & 0.17 & 0.21 & 0.28 & 0.44 & 0.43 \\
\end{tabular}

Donor XXI (saline solution)

\begin{tabular}{lcccccc}
\hline Pulse rate $(\mathrm{bpm})$ & 62 & 100 & 125 & 140 & 152 & 176 \\
\hline Mean pressure $(\mathrm{mmHg})$ & 101.8 & 99.7 & 98.2 & 95.4 & 98.8 & 95.3 \\
Pulse pressure $(\mathrm{mmHg})$ & 37.7 & 39.0 & 34.6 & 38.0 & 39.0 & 38.2 \\
Max $\Delta d(\mathrm{~mm})$ & 1.7 & 1.9 & 1.8 & 1.9 & 1.9 & 2.0 \\
Max $\Delta b(\mathrm{~mm})$ & 0.54 & 0.54 & 0.52 & 0.50 & 0.48 & n.a. \\
$\eta$ diameter change & 0.15 & 0.21 & 0.25 & 0.26 & 0.29 & 0.37 \\
Storage modulus $(\mathrm{kPa})$ & 259 & 260 & 250 & 244 & 235 & 214 \\
$\eta$ bending & 0.22 & n.a. & n.a. & n.a. & n.a. & n.a. \\
\hline \hline
\end{tabular}

(Table continued)
TABLE II. (Continued)

Donor XXI (water/glycerin mix)

\begin{tabular}{lcccccc}
\hline Pulse rate $(\mathrm{bpm})$ & 57 & 94 & 126 & 140 & 161 & 176 \\
\hline Mean pressure $(\mathrm{mmHg})$ & 98.6 & 97.4 & 98.0 & 96.7 & 97.6 & 100.0 \\
Pulse pressure $(\mathrm{mmHg})$ & 35.7 & 38.4 & 36.6 & 38.7 & 37.5 & 37.2 \\
Max $\Delta d(\mathrm{~mm})$ & 1.9 & 1.9 & 1.9 & 1.8 & 1.5 & 1.6 \\
Max $\Delta b(\mathrm{~mm})$ & 0.46 & 0.44 & 0.44 & 0.50 & n.a. & n.a. \\
$\eta$ diameter change & 0.15 & 0.22 & 0.25 & 0.33 & 0.37 & 0.49 \\
Storage modulus $(\mathrm{kPa})$ & 254 & 264 & 244 & 268 & 299 & 268 \\
$\eta$ bending & 0.15 & n.a. & n.a. & n.a. & n.a. & n.a. \\
\hline \hline
\end{tabular}

error was estimated as twice the error incurred from each laser, as two lasers were used in each direction: horizontal and vertical. Therefore, the error was $\pm 0.01 \mathrm{~mm}$ and was not included in Table II to minimize redundancy. Similarly, the mean pressure error was $\pm 1.5 \mathrm{mmHg}$, while the pulse press error was $\pm 0.25 \mathrm{mmHg}$.

\section{APPENDIX B: ADDITIONAL RESULTS ON BENDING VIBRATIONS}

One period of the bending oscillations in the horizontal plane for the aortic segments of donors XVIII and XVI at 126 and 155 bpm, respectively, is presented in Figs. 9(a) and 9 (b) in the time domain. As the pulse rate increases, there are larger contributions of vibrations at higher frequencies. The first bending mode of natural vibrations has a significantly lower frequency in Fig. 9(a) than in Fig. 9(b).

\section{APPENDIX C: DERIVATION OF EQ. (7)}

A hollow cylinder of homogeneous, isotropic, and elastic material is assumed for the aorta specimen. For the case of plane strain and assuming zero displacement in the axial direction, the radial displacement $w$ of an internally pressurized hollow circular cylinder of inner radius $R_{i}$ and exterior radius $R_{e}$ satisfies the equation [34]

$$
\frac{\partial}{\partial r}\left(\frac{\partial w}{\partial r}+\frac{w}{r}\right)=0
$$

where $r$ is the radius of a generic point of the cylinder. The solution of Eq. (C1) is

$$
w=A r+B / r,
$$

where $A$ and $B$ are coefficients to be determined from the boundary conditions. The diameter change of the external surface of the cylinder is denoted by $\Delta d$. Therefore, a radial displacement is given by $\Delta d / 2$. An infinitesimal radial 

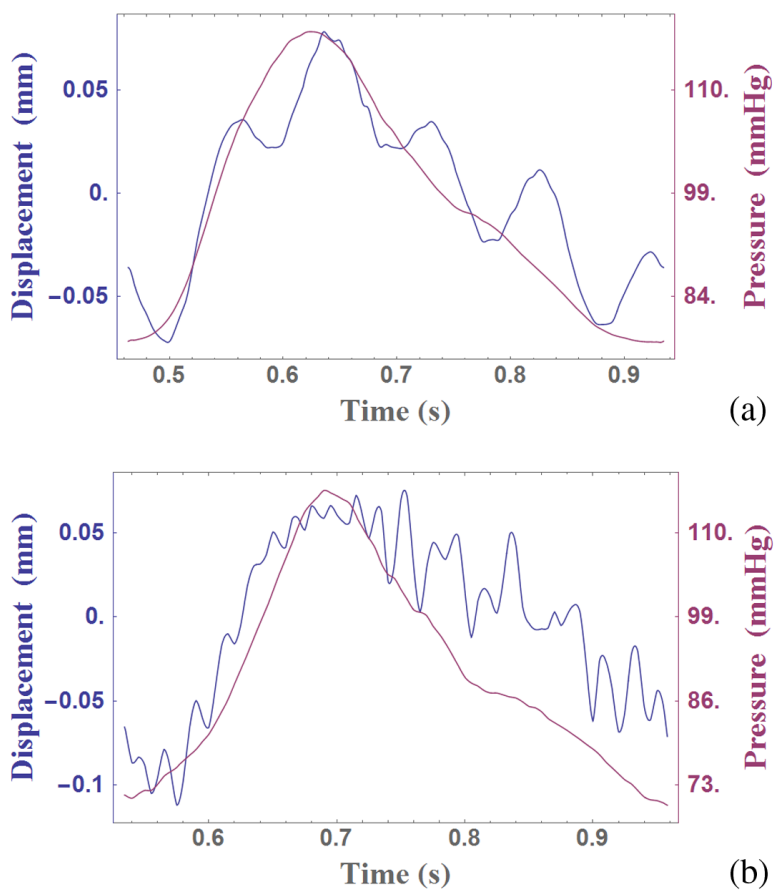

FIG. 9. Bending displacement of one period showing vibrations; red line, pressure; blue line, lateral displacement at midlength of the aortic segment. (a) Donor XVIII at $126 \mathrm{bpm}$ in the horizontal direction, showing vibrations at $10.1 \mathrm{~Hz}$; (b) donor $\mathrm{XVI}$ at $155 \mathrm{bpm}$ in the horizontal direction showing vibrations at $46.4 \mathrm{~Hz}$.

displacement $\mathrm{d} \Delta d / 2$ is assumed at the outer surface of the cylinder, i.e.,

$$
\left.w\right|_{r=R_{e}}=\mathrm{d} \Delta d / 2 .
$$

The radial displacement at the inner surface can be written as

$$
\left.w\right|_{r=R_{i}}=\mathrm{d} \Delta d_{i} / 2 .
$$

In the case of a hollow cylinder of incompressible material, the infinitesimal radial displacement at the inner surface $\mathrm{d} \Delta d_{i} / 2$ can be related to the displacement $\mathrm{d} \Delta d / 2$ by considering that the original volume of the cylinder is unchanged after deformation (note that the length is unchanged by the assumption),

$$
\left(R_{e}+\frac{\mathrm{d} \Delta d}{2}\right)^{2}-\left(R_{i}+\frac{\mathrm{d} \Delta d_{i}}{2}\right)^{2}=R_{e}^{2}-R_{i}^{2}
$$

Equation (C4) gives

$$
\begin{aligned}
\Delta d_{i} & =-2 R_{i}+\sqrt{4 R_{i}^{2}+4 R_{e} \mathrm{~d} \Delta d+\mathrm{d} \Delta d^{2}} \\
& \simeq-2 R_{i}+2 R_{i} \sqrt{1+\frac{R_{e} \mathrm{~d} \Delta d}{R_{i}^{2}}} \simeq \frac{R_{e} \mathrm{~d} \Delta d}{R_{i}},
\end{aligned}
$$

where $R_{i}$ is the inner radius of the aorta and $R_{e}$ is the external radius of the aorta. Approximations in Eq. (C5) are justified by the hypothesis of infinitesimal displacement. By inserting Eq. (C5) into Eq. (C3b), and then solving the system given by Eqs. (C3a) and (C3b), the coefficients $A$ and $B$ are obtained as

$$
\begin{gathered}
A=0, \\
B=R_{e} \mathrm{~d} \Delta d / 2 .
\end{gathered}
$$

The circumferential strain $\varepsilon_{\theta \theta}$ is given by

$$
\varepsilon_{\theta \theta}=\frac{w}{r}=A+\frac{B}{r^{2}}=\frac{R_{e} \mathrm{~d} \Delta d}{2 r^{2}} .
$$

In the present case, there is zero displacement in the axial direction, $\varepsilon_{x x}=0$, which gives

$$
\sigma_{x x}=\nu\left(\sigma_{\theta \theta}+\sigma_{r r}\right)
$$

where $\nu$ is the Poisson coefficient of the material. Therefore, by using Eq. (C8), the circumferential strain $\varepsilon_{\theta \theta}$ can be written as

$$
\begin{aligned}
\varepsilon_{\theta \theta} & =\frac{\sigma_{\theta \theta}}{E}-\frac{\nu}{E}\left(\sigma_{r r}+\sigma_{x x}\right) \\
& =\frac{1}{E}\left[\sigma_{\theta \theta}\left(1-\nu^{2}\right)-\nu(1+\nu) \sigma_{r r}\right],
\end{aligned}
$$

where $E$ is Young's modulus. The radial and circumferential normal stresses are given by [34]

$$
\begin{aligned}
\sigma_{r r} & =p \frac{R_{i}^{2}}{R_{e}^{2}-R_{i}^{2}}\left(1+\frac{R_{e}^{2}}{r^{2}}\right), \\
\sigma_{\theta \theta} & =p \frac{R_{i}^{2}}{R_{e}^{2}-R_{i}^{2}}\left(1-\frac{R_{e}^{2}}{r^{2}}\right),
\end{aligned}
$$

where $p$ is the internal pressure. By combining Eqs. (C9)(C11), and assuming a small variation of pressure, $d p$, that generates a small radial displacement $w$, the following expression is obtained:

$$
E \varepsilon_{\theta \theta}=p \frac{R_{i}^{2}}{R_{e}^{2}-R_{i}^{2}}(1+\nu)\left[(1-2 \nu)+\frac{R_{e}^{2}}{r^{2}}\right] .
$$


Inserting Eq. (C7) into Eq. (C12) and considering that for incompressible material $\nu=0.5$, Young's modulus is obtained as

$$
\begin{aligned}
E & =\frac{2 R_{i}^{2}}{R_{e}} \frac{R_{e}^{2}+(1-2 \nu) r^{2}}{R_{e}^{2}-R_{i}^{2}}(1+\nu) \frac{\mathrm{d} p}{\mathrm{~d} \Delta d} \\
& =\frac{3 R_{i}^{2} R_{e}}{\left(R_{e}^{2}-R_{i}^{2}\right)} \frac{\mathrm{d} p}{\mathrm{~d} \Delta d}=\frac{3 R_{i}^{2} R_{e}}{2 h R_{m}} \frac{\mathrm{d} p}{\mathrm{~d} \Delta d},
\end{aligned}
$$

where $h=R_{e}-R_{i}$ is the thickness of the cylinder wall and $R_{m}=\left(R_{e}+R_{i}\right) / 2$ is the mean radius. Generalizing Eq. (C13) to a nonlinear viscoelastic material, the storage modulus $E^{\prime}$ is given by

$$
E^{\prime}=\frac{3 R_{i}^{2} R_{e}}{2 h R_{m}} \frac{\mathrm{d} p}{\mathrm{~d} \Delta d}
$$

where $\mathrm{d} p / \mathrm{d} \Delta d$ is the slope of the curve representing the pressure versus exterior diameter change. Since the pressure is measured in $\mathrm{mmHg}$ and the diameter change in $\mathrm{mm}$, the conversion factors to $\mathrm{Pa}$ and $\mathrm{m}$ must be considered to present the storage modulus in SI units. Equation (C14) coincides with the one reported by Bergel when the value $\nu=0.5$ is assumed [25,35]. An approximate expression of Eq. (C14) was assumed in Ref. [27] to evaluate the storage moduli of aortas.

\section{APPENDIX D: VISCOELASTIC CHARACTERIZATION OF AORTIC STRIPS}

Uniaxial harmonic tests have been considered as an effective method of characterizing the viscoelastic material properties of the aortic wall in the circumferential and axial directions, both for the complete wall and each layer: intima, media, and adventitia [9,36]. The loss factors and storage moduli obtained with the MCL at $60 \mathrm{bpm}$ were compared to the results of the uniaxial harmonic tests on aortic strips (dimensions of $32 \times 8 \mathrm{~mm}$ ) taken in the circumferential and axial directions, for donors XVI, XVII, and XVIII. The results are presented in Fig. 10. Two harmonic cyclic strain frequencies were considered: 1 and $3 \mathrm{~Hz}$. Although $60 \mathrm{bpm}$ corresponds to $1 \mathrm{~Hz}$, the cyclic pressure in the MCL was far from being a perfect harmonic. From the hysteresis cycles, the loss factor and storage modulus can be identified.

Results from four uniaxial tests for each aorta under physiological applied prestretch are presented. The loading frequencies were 1 and $3 \mathrm{~Hz}$. Two strips of the full wall were tested: axial and circumferential strips, resulting in the four permutations. The experiments were carried out at physiological cyclic strain, $2 \%$ to $8 \%$, depending on the donor's age. It is important to note that the cyclic strain introduced by the pulsatile flow in the MCL is not uniaxial, but it mimics the physiological conditions. In the MCL, the axial and circumferential strains act simultaneously on the
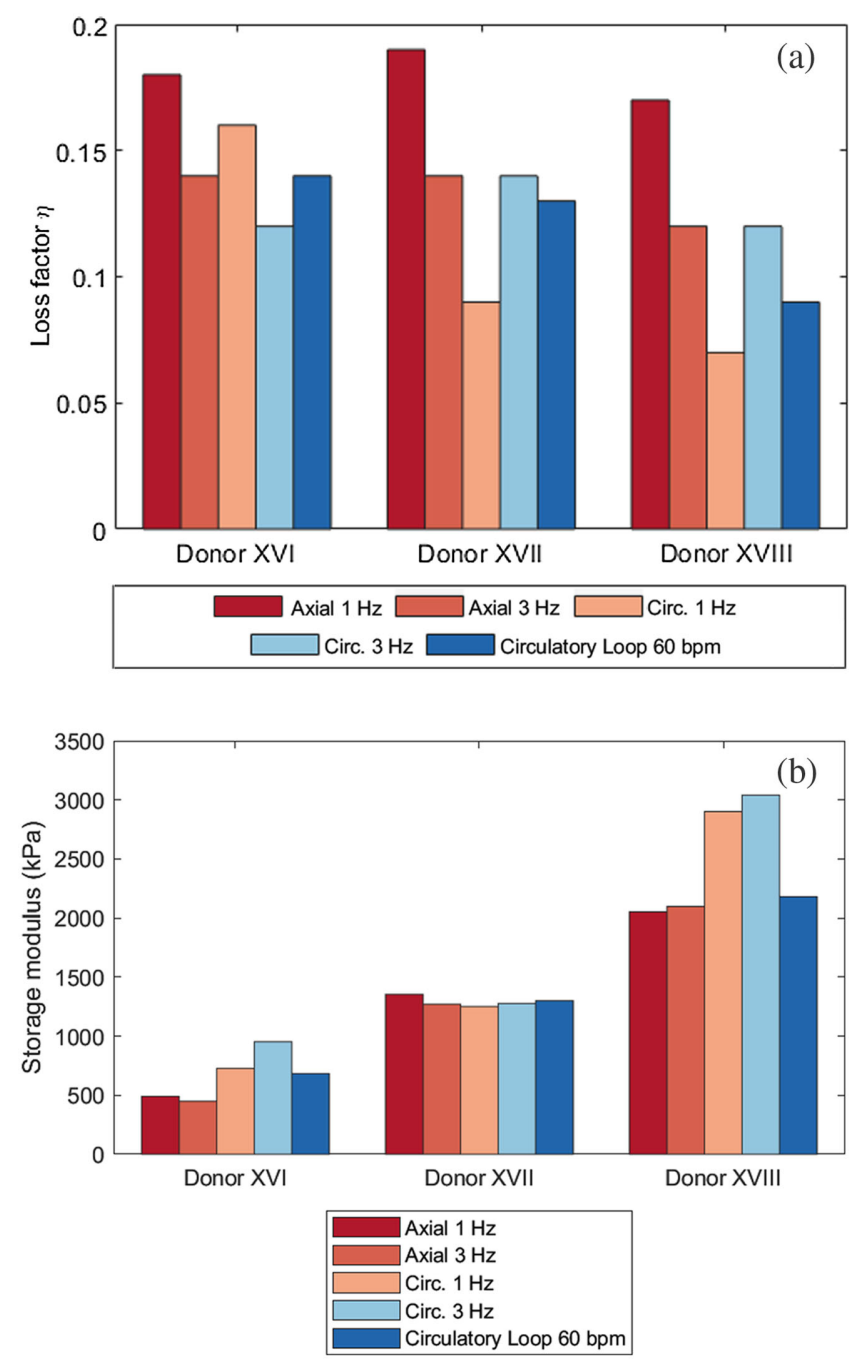

FIG. 10. Comparison of viscoelastic parameters obtained for uniaxial harmonic tests on circumferential and axial strips and in the MCL at around $60 \mathrm{bpm}$ pulse rate. Axial strips: uniaxial harmonic tests at 1 and $3 \mathrm{~Hz}$ at physiological cyclic strain according to donor age (donor XVI $=5 \%$ XVII $=5 \% ; \mathrm{XVIII}=$ $2.5 \%$ in engineering strain) under physiological initial pre-stretch (donor XVI $=1.33 ; \mathrm{XVII}=1.40 ; \mathrm{XVIII}=1.23$ corresponding to about $70 \mathrm{kPa}$ of nominal stress). Circumferential (circ.) strips: uniaxial harmonic tests at 1 and $3 \mathrm{~Hz}$ at physiological cyclic strain according to donor's age (donor XVI $=5 \%$; XVII $=5 \%$; $\mathrm{XVIII}=1.5 \%$ in engineering strain) under physiological initial pre-stretch $\quad($ donor $\quad \mathrm{XVI}=1.59 ; \quad \mathrm{XVII}=2.07 ; \quad \mathrm{XVIII}=1.39$ corresponding to about $70 \mathrm{kPa}$ of nominal stress): (a) loss factor; (b) storage modulus. maroon box, axial $1 \mathrm{~Hz}$; dark brown box, axial $3 \mathrm{~Hz}$; light brown box, circ. $1 \mathrm{~Hz}$; light blue box, circ. $3 \mathrm{~Hz}$; dark blue box, circulatory loop at $60 \mathrm{bpm}$.

specimen, whereas in the uniaxial harmonic tests the strain is applied in a single direction. This method can induce significant differences in the viscoelastic behavior [19]. Therefore, this comparison of donors XVI, XVII, and XVIII also aimed to assess whether uniaxial harmonic tests were an effective substitute for the MCL. 
The results presented in Fig. 10(a) show that the loss factors obtained in the MCL at 60-bpm pulse rate for these three aortas are consistent with the data obtained from the uniaxial harmonic tests. Slight variations were observed for the loss factors found from the uniaxial tests between the values at 1 and $3 \mathrm{~Hz}$ in both directions. However, these differences were not very significant. For donors XVII and XVIII, the loss factor value in the MCL at $60 \mathrm{bpm}$ was in between the axial and circumferential loss factors found at $1 \mathrm{~Hz}$. Instead, for donor XVI, the loss factor from the MCL at $60 \mathrm{bpm}$ was slightly below the two from the uniaxial traction test at $1 \mathrm{~Hz}$. This result reinforces the fact that the flow contributed little to the dissipative behavior of the aortic segment at low pulse rates. Therefore, the flow-aorta coupling and dynamics at increased pulse rates must have accounted for the larger loss factor values obtained in the MCL, as explained in the paper.

Conversely, the storage modulus showed a strong dependence to the type of experiment conducted to measure it. The value of the modulus was shown to vary very significantly with the applied prestretch, to the point that almost any value larger than a threshold can be obtained by selecting the proper prestretch. However, since the strain is bidimensional in physiological conditions, it is very tricky to select a representative prestretch. Here, the prestretch giving results closer to the MCL was chosen between the two predetermined possible physiological values. Results are shown in Fig. 10(b). For donors XVI and XVII, similar storage modulus values were found for both the MCL and the uniaxial harmonic traction tests. Instead, for donor XVIII, the applied prestretch resulted in vastly different storage moduli values. This result is one of the clear drawbacks of the uniaxial traction test experiment, a level of prestretch must be presumed. Without prior knowledge, it is impossible to know the correct amount of applied prestretch for the uniaxial harmonic test. In the case of donor XVIII, the necessary prestretch was in the range of 1.29 since the axial values were much closer to the ones obtained with the MCL, but this could not have been known without the MCL experiment, further corroborating the importance of this novel MCL experiment for viscoelastic aortic material characterizations.

[1] O. Khavjou, D. Phelps, and A. Leib, Projections of Cardiovascular Disease Prevalence and Costs: 20152035, Technical Report prepared for the American Heart Association, RTI International, 2016.

[2] E. S. Place, N. D. Evans, and M. M. Stevens, Complexity in Biomaterials for Tissue Engineering, Nat. Mater. 8, 457 (2009).

[3] D. Wang, L. Vahala, and Z. Hao, Radial and Longitudinal Motion of the Arterial Wall: Their Relation to Pulsatile Pressure and Flow in the Artery, Phys. Rev. E 98, 032402 (2018).
[4] M. Amabili, P. Balasubramanian, I. D. Breslavsky, G. Ferrari, and E. Tubaldi, Viscoelastic Characterization of Woven Dacron for Aortic Grafts by Using DirectionDependent Quasi-Linear Viscoelasticity, J. Mech. Behav. Biomed. Mater. 82, 282 (2018).

[5] G. Ferrari, P. Balasubramanian, E. Tubaldi, F. Giovanniello, and M. Amabili, Experiments on Dynamic Behaviour of a Dacron Aortic Graft in a Mock Circulatory Loop, J. Biomech. 86, 132 (2019).

[6] E. Ehret, K. Bircher, A. Stracuzzi, V. Marina, M. Zündel, and E. Mazza, Inverse Poroelasticity as a Fundamental Mechanism in Biomechanics and Mechanobiology, Nat. Commun. 8, 1002 (2017).

[7] T. M. Morrison, G. Choi, C. K. Zarins, and C. A. Taylor, Circumferential and Longitudinal Cyclic Strain of the Human Thoracic Aorta: Age-Related Changes, J. Vasc. Surg. 49, 1029 (2009).

[8] C. J. Mills, I. T. Gabe, J. H. Gault, D. T. Mason, J. Ross, E. Braunwald, and J. P. Shillingford, Pressure-Flow Relationships and Vascular Impedance in Man, Cardiovasc. Res. 4, 405 (1970).

[9] M. Amabili, P. Balasubramanian, I. Bozzo, I. D. Breslavsky, and G. Ferrari, Layer-Specific Hyperelastic and Viscoelastic Characterization of Human Descending Thoracic Aortas, J. Mech. Behav. Biomed. Mater. 99, 27 (2019).

[10] C. E. Taylor, Z. W. Dziczkowski, and G. E. Miller, Automation of the Harvard Apparatus Pulsatile Blood Pump, J. Med. Devices 6, 045002 (2012).

[11] C. E. Taylor and G. E. Miller, Implementation of an Automated Peripheral Resistance Device in a Mock Circulatory Loop with Characterization of Performance Values Using Simulink Simscape and Parameter Estimation, J. Med. Devices 6, 045001 (2012).

[12] C. E. Taylor and G. E. Miller, Mock Circulatory Loop Compliance Chamber Employing a Novel Real-Time Control Process, J. Med. Devices 6, 045003 (2012).

[13] D. Valdez-Jasso, D. Bia, Y. Zócalo, R. L. Armentano, M. A. Haider, and M. S. Olufsen, Linear and Nonlinear Viscoelastic Modeling of Aorta and Carotid Pressure-Area Dynamics under In Vivo and Ex Vivo Conditions, Annals Biomed. Eng. 39, 1438 (2011).

[14] D. Valdez-Jasso, M. A. Haider, H. T. Banks, D. B. Santana, Y.Z. Germán, R. L. Armentano, and M. S. Olufsen, Analysis of Viscoelastic Wall Properties in Ovine Arteries, IEEE Trans. Biomed. Eng. 56, 210 (2009).

[15] R. L. Armentano, J. G. Barra, F. M. Pessana, D. O. Craiem, S. Graf, D. B. Santana, and R. A. Sanchez, Smart Smooth Muscle Spring-Dampers. Smooth Muscle Smart Filtering Helps to More Efficiently Protect the Arterial Wall, IEEE Eng. Med. Biol. Mag. 26, 62 (2007).

[16] R. L. Armentano, J. G. Barra, J. Levenson, A. Simon, and R. H. Pichel, Arterial Wall Mechanics in Conscious Dogs. Assessment of Viscous, Inertial, and Elastic Moduli to Characterize Aortic Wall Behavior, Circ. Res. 76, 468 (1995).

[17] M. Amabili, Nonlinear Mechanics of Shells and Plates in Composite, Soft and Biological Materials (Cambridge University Press, New York, 2018).

[18] G. A. Holzapfel, T. C. Gasser, and M. A. Stadler, A Structural Model for the Viscoelastic Behavior of Arterial Walls: 
Continuum Formulation and Finite Element Analysis, Eur. J. Mech. A 21, 441 (2002).

[19] R. H. Dhume and V.H. Barocas, Emergent StructureDependent Relaxation Spectra in Viscoelastic Fiber Networks in Extension, Acta Biomater. 87, 245 (2019).

[20] H. Weisbecker, D. M. Pierce, P. Regitnig, and G. A. Holzapfel, Layer-Specific Damage Experiments and Modeling of Human Thoracic and Abdominal Aortas with NonAtherosclerotic Intimal Thickening, J. Mech. Behav. Biomed. Mater. 12, 93 (2012).

[21] J. A. Niestrawska, C. Viertler, P. Regitnig, T. U. Cohnert, G. Sommer, and G. A. Holzapfel, Microstructure and Mechanics of Healthy and Aneurysmatic Abdominal Aortas: Experimental Analysis and Modelling, J. R. Soc. Interface 13, 20160620 (2016).

[22] J. Schriefl, G. Zeindlinger, D. M. Pierce, P. Regitnig, and G. A. Holzapfel, Determination of the Layer-Specific Distributed Collagen Fibre Orientations in Human Thoracic and Abdominal Aortas and Common Iliac Arteries, J. R. Soc. Interface 9, 1275 (2012).

[23] G. A. Holzapfel, J. A. Niestrawska, R. W. Ogden, A. J. Reinisch, and A.J. Schriefl, Modelling Non-Symmetric Collagen Fibre Dispersion in Arterial Walls, J. R. Soc. Interface 12, 20150188 (2015).

[24] G. A. Holzapfel, G. Sommer, C. T. Gasser, and P. Regitnig, Determination of Layer-Specific Mechanical Properties of Human Coronary Arteries with Nonatherosclerotic Intimal Thickening and Related Constitutive Modeling, Am. J. Physiol. Heart Circ. Physiol. 289, H2048 (2005).

[25] D. H. Bergel, The Dynamic Elastic Properties of the Arterial Wall, J. Physiol. 156, 458 (1961).
[26] E.-J. Courtial, L. Fanton, M. Orkisz, P. C. Douek, L. Huet, and R. Fulchiron, Hyper-Viscoelastic Behavior of Healthy Abdominal Aorta, IRBM 37, 158 (2016).

[27] T. Imura, K. Yamamoto, T. Satoh, K. Kanamori, T. Mikami, and H. Yasuda, In Vivo Viscoelastic Behavior in the Human Aorta, Circ. Res. 66, 1413 (1990).

[28] L. Horný, M. Netušil, and T. Voňavková, Axial Prestretch and Circumferential Distensibility in Biomechanics of Abdominal Aorta, Biomech. Model. Mechanobiol. 13, 783 (2014).

[29] C. A. J. Schulze-Bauer, C. Mörth, and G. A. Holzapfel, Passive Biaxial Mechanical Response of Aged Human Iliac Arteries, J. Biomech. Eng. 125, 395 (2003).

[30] Y. C. Fung, Biomechanics: Circulation, 2nd ed. (Springer, New York, 1997).

[31] R. H. Mohiaddin, P. J. Kilner, S. Rees, and D. B. Longmore, Magnetic Resonance Volume Flow and Jet Velocity Mapping in Aortic Coarctation, J. Am. Col. Cardiol. 22, 1515 (1993).

[32] E. Tubaldi, M. P. Païdoussis, and M. Amabili, Nonlinear Dynamics of Dacron Aortic Prostheses Conveying Pulsatile Flow, J. Biomech. Eng. 140, 061004 (2018).

[33] M. E. Safar, Arterial Stiffness as a Risk Factor for Clinical Hypertension, Nat. Rev. Cardiol. 15, 97 (2017).

[34] A. E. H. Love, A Treatise on the Mathematical Theory of Elasticity (Dover, New York, 1927).

[35] D. H. Bergel, The Static Elastic Properties of the Arterial Wall, J. Physiol. 156, 445 (1961).

[36] M. Amabili, P. Balasubramanian, and I. D. Breslavsky, Anisotropic Fractional Viscoelastic Constitutive Models for Human Descending Thoracic Aortas, J. Mech. Behav. Biomed. Mater. 99, 186 (2019). 